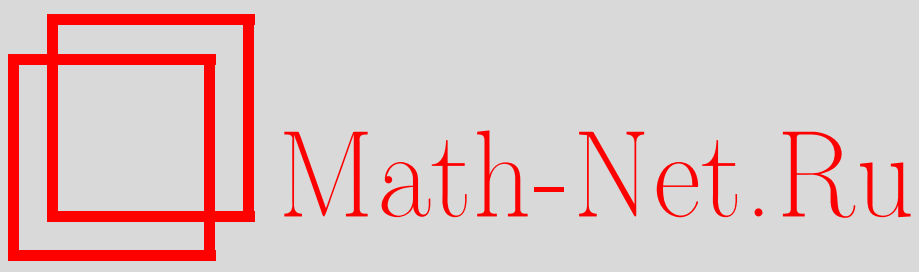

В. С. Губа, О связи проблем равенства и делимости слов для полугрупп с одним определяющим соотношением, Изв. РАН. Сер. матем., 1997, том 61, выпуск 6, 2758

DOI: https://doi.org/10.4213/im167

Использование Общероссийского математического портала Math-Net.Ru подразумевает, что вы прочитали и согласны с пользовательским соглашением

http://www.mathnet.ru/rus/agreement

Параметры загрузки:

IP : 54.80 .73 .141

26 апреля 2023 г., 10:32:45 
УДК 519.4

\author{
B. С. Губо
}

\title{
О связи проблем равенства и делимости слов для полугрупп с одним определяющим соотношением
}

\begin{abstract}
В статье получен ряд результатов, касающихся связи проблем равенства и делимости слов для полугрупп с одним определяющим соотношением. В частности, одним из основных результатов работы является теорема о равносильности трех алгоритмических проблем: равенства, левой делимости и правой делимости для полугрупп, заданных соотношением вида $a=b Q a$, где $Q$ есть слово от $a, b$.

Библиографиия: 25 наименований.
\end{abstract}

Проблема равенства слов для полугрупп с одним определяюшим соотношением остается пока открытой. Усилиями С.И. Адяна и его учеников она сведена к случаю полугрупп, задаваемых с помощью двух порождающих, $a$ и $b$, и соотношения, имеюшего либо вид $a=b Q a$, либо вид $a P a=b Q a$, где $P, Q$ - некоторые слова от $a, b$, возможно, пустые. (См. по этому поводу работы [3], [6], [7], [11], [12] и уточнение в [5]. Несколько более слабый результат содержится в [21].)

Из [4] известно, что проблема равенства для полугрупп, заданных соотношениями без левых (правых) циклов, сводится к проблеме левой (правой) делимости слов для данной полугруппы. В частности, этот результат применим к обоим случаям, отмеченным вьше.

В настоящей работе анализируется прежде всего случай полугрупп, заданных соотношением вида $a=b Q a$. Отметим, что проблемы равенства и левой делимости слов для таких полугрупп некоторое время считались решенными. Однако в [5] отмечено, что доказательство опирается на основной результат статьи [17], которая является ошибочной. Заметим, что в [11] доказана разрешимость обеих проблем для случая, когда $b Q$ есть истинная степень некоторого слова в свободной полугруппе.

Обрисуем вкратце основные результаты настоящей работы. В $\S 1$ мы доказываем лемму о моноидах, удовлетворяющих закону левостороннего сокращения. (Таковы, в частности, все полугрупшы с единицей, задаваемые копредставлением без левых циклов, в том числе полугруппы с одним соотношением любого из видов $a=b Q a$ или $a P a=b Q a$.) Мы сводим проблему равенства слов для таких полугрупп к проблеме вхождения слов в так называемое полунормальное замыкание множества слов в свободной групе. Этот результат будет использован в дальнейшем. Думается, что он представляет и самостоятельный интерес.

В $\S 2$ мы сводим проблему левой делимости для полугрупп с одним соотношением вида $a=b Q a$ к проблеме вхождения в конечно порожденную подполугруппу в группе с одним определяюшим соотношением. Заметим, что эта группа не всегда

Работа выполнена при поддержке РФФИ, грант 1541, а также ISF, грант MID 000.

$$
\text { (C) В.С. ГУБА } 1997
$$


задается соотношением $b Q=1$. Результат по своей форме (но не по методу доказательства) напоминает результаты Микина и Марголиса (устное сообщение), относящиеся к инверсным полугруппам.

В качестве применения нашего результата мы анализируем случай полугрупп $\langle a, b \mid a=b Q a\rangle$, для которых соответствуюшая группа с одним определяющим соотношением оказывается свободной. Поскольку проблема вхождения в конечно порожденную полугруппу разрешима для свободной группы [19], мы получаем разрешимость проблемы левой делимости (а потому и проблемы слов) для ряда случаев, охватываюших, в частности, ситуацию, когда $b Q$ есть истинная степень.

В $\S 3$ доказьвается, что для полугрупп с соотношением вида $a=b Q a$ проблема равенства слов на самом деле эквивалентна проблеме левой делимости. Это, по всей видимости, позволяет сделать вывод, что решение проблемы слов для данных полугрупп неизбежно влечет исследование некоторых вопросов типа проблемы вхождения в конечно порожденную подполугруппу в группах с одним определяюшим соотношением. Кроме того, мы доказываем, что проблема равенства слов может быть сведена к проблеме равенства данного слова порождаюшему $a$.

$\S 4$ посвящен проблеме правой делимости для рассматриваемых полугрупп. Надо отметить, что этот вопрос не исследовался в цитированных выше работах. Мы доказываем, что данная проблема также эквивалентна проблеме левой делимости (и проблеме равенства слов). Особую роль играет проблема делимости справа порождающего $a$ на заданное слово.

Наконец, в $\S 5$ мы исследуем вопросы, относяшиеся к полугруппам, заданным соотношениями без левых (правых) циклов. Эти вопросы идейно связаны с тематикой работы, хотя и не имеют непосредственного отношения к ее заглавию. Тем не менее мы считаем уместным дать здесь некоторые (структурно-алгебраические) условия для полугрупп, необходимо вьполняюшиеся для полугрупп без односторонних циклов. Это, в частности, продолжает результаты из [12] - статьи с той же тематикой, что и наша.

Автор благодарит за ценные обсуждения результатов данной статьи С. И. Адяна, С. Марголиса, Дж. Микина, Г.У. Оганесяна, М. Сапира, А. Ю. Ольшанского, С. Прайда.

\section{§ 1. Моноиды с левосторонним сокращением}

Символ $\equiv$ будет обозначать графическое равенство слов. Длину слова $X$ мы обозначаем через $|X|$.

Напомним, что полугрупп $S$ удовлетворяет закону левостороннего сокращения, если для любых $x, y, z \in S$ из условия $x y=x z$ следует, что $y=z$.

Мы будем рассматривать моноиды (полугруппы с единицей), заданные своим копредставлением вида

$$
\left\langle x_{1}, \ldots, x_{n} \mid A_{i}=B_{i}, i \in I\right\rangle,
$$

где $A_{i}, B_{i}$ - произвольные слова, возможно, пустые. (Множество порождающих для простоты предполагается конечным.)

Хорошо известен факт, что если полугруппа с единицей $S$ задана копредставлением (1) без левых циклов, то она удовлетворяет закону левостороннего сокрашения. 
Пусть $F_{n}$ - свободная группа с множеством свободных порождаюших $x_{1}, \ldots, x_{n}$. Рассмотрим произвольное множество $P \subseteq F_{n}$. Назовем полунормальнылм замыканием множества $P$ множество элементов, равных в $F_{n}$ какому-либо из слов вида

$$
Z_{1}^{-1} P_{1}^{ \pm 1} Z_{1} \ldots Z_{k}^{-1} P_{k}^{ \pm 1} Z_{k}
$$

где $P_{1}, \ldots, P_{k} \in P$, а $Z_{1}, \ldots, Z_{k}$ - позитивные слова (т.е. слова без отрицательных степеней букв) над алфавитом $X=\left\{x_{1}, \ldots, x_{n}\right\}$.

Ясно, что полунормальное замыкание множества $P$ будет подгруппой в $F_{n}$.

Отметим, что идея полунормального замыкания встречалась в работе [23], но там речь шла не о свободной группе, а о полициклическом моноиде.

ЛЕмма 1.1. Пусть $S$ - полугруппа с единицей, заданная копредставлением $в и \partial а$

$$
\left\langle X \mid A_{i}=B_{i}, i \in I\right\rangle
$$

Предположим, что в $S$ выполнен закон левостороннего сокращения. Обозначим через $F$ свободную группу с базой $X$, и пусть $P$ - множество слов вида $A_{i}^{-1} B_{i}(i \in I)$, а $\bar{P}$ - полунормальное замыкание $P$ в $F$.

Утверждается, что два позитивных слова, $U$ и $V$, над алфавитом $X$ равны в $S$ тогда и только тогда, когда әлемент $U^{-1} V$ принадлежит $\bar{P}$.

ДокАЗАТЕЛЬСТво. Предположим, что $U$ и $V$ равны в $S$. Это означает по определению, что существуют слова $W_{0}, \ldots, W_{m}$ такие, что $U \equiv W_{0}, W_{m} \equiv V$, и для любого $j$ от 1 до $m$ можно записать $W_{j-1} \equiv C D E, W_{j} \equiv C F E$, где либо $D \equiv A_{i}, F \equiv B_{i}$, либо $D \equiv B_{i}, F \equiv A_{i}$. Тогда $U^{-1} V \equiv W_{0}^{-1} W_{m}=$ $\left(W_{0}^{-1} W_{1}\right) \ldots\left(W_{m-1}^{-1} W_{m}\right)$, однако $W_{j-1}^{-1} W_{j}=E^{-1}\left(D^{-1} F\right) E$, что равно $E^{-1} R_{i}^{ \pm 1} E$, где $R_{i}$ есть $A_{i}^{-1} B_{i}$. Таким образом, $U^{-1} V \in \bar{P}$.

Установим обратное. Пусть $U^{-1} V \in \bar{P}$, где $U, V$ - позитивные слова. Тогда в свободной группе имеет место равенство $U^{-1} V=Z_{1}^{-1} P_{1}^{ \pm 1} Z_{1} \ldots Z_{k}^{-1} P_{k}^{ \pm 1} Z_{k}$, где слова $Z_{1}, \ldots, Z_{k}$ позитивные, а $P_{1}, \ldots, P_{k} \in\left\{A_{i}^{-1} B_{i} \mid i \in I\right\}$.

Всякое слово вида $Z^{-1}\left(A_{i}^{-1} B_{i}\right)^{ \pm 1} Z$ имеет вид $C^{-1} D$, где $C, D$ - позитивные слова, причем $C$ равно $D$ в $S$, так как слова $A_{i} Z$ и $B_{i} Z$ равны в $S$. Поэтому $U^{-1} V$ равно в $F$ слову вида $C_{1}^{-1} D_{1} \ldots C_{l}^{-1} D_{l}$, где $C_{j}$ равно $D_{j}$ в $S(1 \leqslant j \leqslant l)$, причем $l$ выберем минимально возможным. При данном $l$ потребуем, чтобы сумма длин всех слов $C_{j}, D_{j}$ была минимальной.

Наша цель - доказать, что $l=1$. Отсюда следует то, что нам необходимо, поскольку тогда $U^{-1} V=C_{1}^{-1} D_{1}$ в группе $F$, где в правой части отсутствуют сокращения, а потому $U \equiv W C_{1}, V \equiv W D_{1}$ для некоторого слова $W$. Поскольку $C_{1}$ и $D_{1}$ равны в $S$, то $U$ и $V$ также равны, что и требовалось доказать.

Итак, предположим, что $l>1$.

Допустим, что одно из слов $C_{2}, \ldots, C_{l}, D_{1}, \ldots, D_{l-1}$ пустое. Если это $C_{j}$, то $C_{j-1}^{-1} D_{j-1} C_{j}^{-1} D_{j} \equiv C_{j-1}^{-1}\left(D_{j-1} D_{j}\right)$, и можно удалить $C_{j}, D_{j}$, заменяя $D_{j-1}$ на $D_{j-1} D_{j}$ и уменьшая при этом $l$. Ясно, что $C_{j-1}$ равно $D_{j-1} D_{j}$ в $S$, так что все условия вьполнены. Аналогично, если пусто $D_{j}$, то

$$
C_{j}^{-1} D_{j} C_{j+1}^{-1} D_{j+1} \equiv\left(C_{j+1} C_{j}\right)^{-1} D_{j+1}
$$


и нужно удалить $C_{j}, D_{j}$, заменяя $C_{j+1}$ на $C_{j+1} C_{j}$. Заметим, что $C_{j+1} C_{j}$ равно $D_{j+1}$ в $S$, и $l$ вновь можно уменьшить.

Итак, все слова (кроме, возможно, $C_{1}$ и $D_{l}$ ) непустые. Допустим, что при некотором $j$ слово $C_{j}^{-1} D_{j}$ сократимо в свободной группе. Тогда $C_{j} \equiv x C^{\prime}, D_{j} \equiv x D^{\prime}$, где $x$ - буква. Заменим $C_{j}$ на $C^{\prime}$, а $D_{j}$ на $D^{\prime}$. Так как в $S$ выполнен закон левостороннего сокрашения, то $C^{\prime}$ и $D^{\prime}$ равны в $S$. При этом уменьшается сумма длин всех слов.

Если ни одно из слов $C_{2}, \ldots, C_{l}, D_{1}, \ldots, D_{l-1}$ не сократится полностью в произведении, то приведенная форма в левой части не будет равна в свободной группе слову вида $U^{-1} V$. Стало быть, при некотором $j$ в произведении $D_{j-1} C_{j}^{-1}$ одно из слов должно полностью сократиться. Рассмотрим два случая.

Если $D_{j-1}$ сократится полностью, то $C_{j} \equiv W D_{j-1}$ при некотором $W$. В этом случае $C_{j-1}^{-1} D_{j-1} C_{j}^{-1} D_{j}$ равно в $F$ слову $C_{j-1}^{-1} W^{-1} D_{j} \equiv\left(W C_{j-1}\right)^{-1} D_{j}$. Удалим $C_{j-1}, D_{j-1}$, заменяя $C_{j}$ на $W C_{j-1}$. Ясно, что $W C_{j-1}$ равно в $S$ слову $W D_{j-1} \equiv$ $C_{j}$, что равно $D_{j}$ в $S$, т.е. получится, что $l$ можно уменьшить на единицу.

Во втором случае, если $C_{j}$ полностью сократилось, т.е. $D_{j-1} \equiv W C_{j}$, имеем, что $C_{j-1}^{-1} D_{j-1} C_{j}^{-1} D_{j}$ равно в $F$ слову $C_{j-1}^{-1} W D_{j}$, и можно удалить $C_{j}, D_{j}$, заменяя $D_{j-1}$ на $W D_{j}$. Очевидно, что $C_{j-1}$ равно в $S$ слову $D_{j-1} \equiv W C_{j}$, что равно $W D_{j}$ в $S$, т.е. вновь можно уменьшить $l$ на единицу. Получено противоречие. Лемма доказана.

СлЕДСТВИЕ 1.1. Проблема равенства слов для полугрупп с одним определяющим соотношением сводится $к$ проблеме вхождения в полунормальное замыкание одного слова в свободной группе с двумя порождающими.

Для доказательства заметим, что моноиды $\langle a, b \mid a=b Q a\rangle,\langle a, b| a P a=$ $b Q a\rangle$ заданы копредставлениями без левых циклов, а потому для этих моноидов выполнен закон левостороннего сокращения. К случаям таких полугрупп сводится проблема равенства для полугрупп с одним соотношением (см. [7] и уточнение в [5] по поводу полугрупп первого вида).

Таким образом, мы имеем проблему вхождения в полунормальное замыкание либо слова $a^{-1} b Q a$, либо слова $a^{-1} P^{-1} a^{-1} b Q a$.

Отметим еше одно важное следствие леммы 1.1. Для начала укажем некоторое очевидное свойство диаграмм над любым полугрупповым копредставлением. Необходимые сведения о полугрупповых диаграммах могут быть найдены в [8], [24], [20].

Пусть имеется диаграмма $\Delta$ над некоторым полугрупповым копредставлением вида (1), задаюшим моноид $S$, и пусть $\alpha, \omega$ - начальная и конечная вершины диаграммы $\Delta$ соответственно. Для любой вершины $v$ диаграммы рассмотрим какие-либо пути $p_{1}, p_{2}$ из $\alpha$ в $v$. Ясно, что метки этих путей равны друг другу в $S$, и тем самым определен элемент $l(v) \in S$, заданный этими метками. Аналогичное утверждение верно для любых путей $q_{1}, q_{2}$ из $v$ в $\omega$, что позволяет определить элемент $r(v) \in S$.

СлЕДСТВИЕ 1.2. Пусть дано произвольное полугрупповое копредставление, и пусть $S$ - заданная с его помощью полугруппа с единицей. Допустим, что в $S$ выполнен закон левостороннего сокращения. Пусть $\Delta$ - некоторая диаграмма над данным копредставлением. Предположим, что для некоторых двух вершин, $v$ и $w$, диаграммы $\Delta$ имеется путь $p$, соединяющий эти 
вершины (в р могут входить как положстельные, так и отрицательные ребра), причем метка пути р равна единице в свободной группе.

Тогда әлементы $r(v)$ и $r(w)$ равны.

(Легко понять, что закон левостороннего сокращения является необходимым условием.)

ДокАЗАТЕЛЬСтво. Представим $p$ в виде $p_{1} q_{1}^{-1} p_{2} q_{2}^{-1} \ldots, p_{k} q_{k}^{-1}$, где пути $p_{1}, \ldots$ $\ldots, p_{k}, q_{1}, \ldots, q_{k}$ состоят только из положительных ребер. Обозначим через $u_{i}$ конец пути $p_{i}$, являющийся также конщом пути $q_{i}(1 \leqslant i \leqslant k)$. Пусть $P_{1}, \ldots, P_{k}$, $Q_{1}, \ldots, Q_{k}$ - метки путей $p_{1}, \ldots, p_{k}, q_{1}, \ldots, q_{k}$ соответственно, а $U_{i}(1 \leqslant i \leqslant k)-$ метка какого-либо пути из $u_{i}$ в заключительную вершину диаграммы $\Delta$. Также пусть $V, W$ - метки каких-либо путей, идущих из $v, w$ соответственно в заключительную вершину (можно считать, что $V \equiv P_{1} U_{1}, W \equiv Q_{k} U_{k}$ ).

Мы имеем следующие равенства в $S$ :

$$
\begin{gathered}
V=P_{1} U_{1}, \quad Q_{1} U_{1}=P_{2} U_{2}, \\
Q_{2} U_{2}=P_{3} U_{3}, \quad \ldots, \quad Q_{k-1} U_{k-1}=P_{k} U_{k}, \\
Q_{k} U_{k}=W .
\end{gathered}
$$

Применим лемму 1.1. Обозначая через $\mathscr{M}$ полунормальное замыкание множества определяюших соотношений в свободной группе, мы имеем

$$
V^{-1} P_{1} U_{1}, \quad U_{1}^{-1} Q_{1}^{-1} P_{2} U_{2}, \quad \ldots, \quad U_{k-1}^{-1} Q_{k-1}^{-1} P_{k} U_{k}, \quad U_{k}^{-1} Q_{k}^{-1} W \in \mathscr{M} .
$$

Так как $\mathscr{M}$ - подгруппа, то произведение всех этих элементов также принадлежит $\mathscr{M}$. Но оно равно в свободной группе слову $V^{-1}\left(P_{1} Q_{1}^{-1} P_{2} Q_{2}^{-1} \ldots P_{k} Q_{k}^{-1}\right) W$, т.е. также и слову $V^{-1} W$, поскольку слово в скобках равно 1 . Следовательно, $V^{-1} W \in \mathscr{M}$, и, вновь применяя лемму 1.1 , получаем, что $V$ и $W$ равны в $S$, что и требовалось доказать.

(Г. У. Оганесян обратил внимание автора на возможность прямого доказательства следствия 1.2 без использования леммы 1.1.)

Нам будет нужно еще одно утверждение (следствие 1.3), которое можно считать известным (ср. [17, лемма 9]).

Рассмотрим полугруппы с единицей, заданные следующим образом. Их порождающие - это буквы $x_{1}, \ldots, x_{n}$, а определяющие соотношения имеют вид

$$
x_{i}=W_{i}\left(x_{1}, \ldots, x_{n}\right), \quad 1 \leqslant i \leqslant m,
$$

при некотором $m \leqslant n$, где $W_{i}$ - произвольные слова от $x_{1}, \ldots, x_{n}$. Положим $P \rightarrow Q$, если при некоторых $C, D$ и некотором $i$ от 1 до $m$ имеем $P \equiv C x_{i} D$, $Q \equiv C W_{i} D$. Обозначим через $\stackrel{*}{\rightarrow}$ реф̆лексивное транзитивное замыкание отношения $\rightarrow$.

Пусть $S$ - полугруппа с единицей, заданная при помощи (3). Нетрудно доказать, что два произвольных слова, $U$ и $V$, равны в $S$ тогда и только тогда, когда существует такое слово $W$, что $U \stackrel{*}{\rightarrow} W, V \stackrel{*}{\rightarrow} W$. (Это легко установить с помошью индукции по длине вывода, связываюшего $U$ и $V$.) 
Пусть $M$ - произвольный моноид, заданньй с помошью (1). Скажем, что слова $U, V$ сравнимы слева в $M$, если существуют слова $U^{\prime}, V^{\prime}$ такие, что $U U^{\prime}$ равно $V V^{\prime}$ в $S$.

Имея полугруппу с единицей $S$, заданную соотношениями вида (3), легко понять, в каких случаях слова $U, V$ в ней будут сравнимы (слева или справа). Допустим, что $U U^{\prime}$ равно $V V^{\prime}$ в $S$, и пусть $W$ - такое слово, что $U U^{\prime} \stackrel{*}{\rightarrow} W, V V^{\prime} \stackrel{*}{\rightarrow} W$. Ясно, что $U \stackrel{*}{\rightarrow} X, V \stackrel{*}{\rightarrow} Y$, где $X, Y$ - некоторые начала $W$. Отсюда очевидно, что если слова $U$ и $V$ сравнимы в $S$ слева, то одно из них делится слева на другое. (Аналогичное утверждение имеет место и для сравнимости справа.)

Для удобства ссылок сформулируем

СлЕДСТВИЕ 1.3. Пусть полугруппа с единичей $S$ задана при помощи (3). Если слова $U, V$ сравнимь в $S$ слева, то либо $U$ делится слева на $V$, либо $V$ делится слева на $U$.

Данное следствие в дальнейшем будет неоднократно использоваться при анализе полугрупп вида $\langle a, b \mid a=b Q a\rangle$, которые, очевидно, удовлетворяют этим условиям.

\section{§ 2. Сведение проблемы делимости к проблеме вхождения}

Пусть $\Pi=\langle a, b \mid a=b Q a\rangle$. Обозначим через $S$ полугруппу, порожденную в П всеми (непустыми) концами слова $b Q a$. По существу такая полугруппа рассматривается в [12]. Там же указан алгоритм, позволяюший эффективно задать копредставление для $S$. Более того, в [12] получен весьма нетривиальный результат о том, что $S$ может быть задана соотношениями как без левых, так и без правых циклов. Проблема левой делимости для П сведена в [12] к проблеме левой делимости для $S$.

(Напомним, что в статье [17], как отмечалось в [5], предпринята неудачная попытка доказать разрешимость проблем равенства и делимости для полугрупп без циклов. Отметим, что, на наш взгляд, эта проблема, по всей видимости, еше сложнее, чем проблема слов для полугрупп с одним определяюшим соотношением.)

Итак, полугруппа $S$ задается соотношениями без циклов. Следовательно, ввиду результата работы [3] $S$ может быть изоморфно вложена в группу. Это означает, что $S$ вложима изоморфно в группу $G$, заданную тем же копредставлением, что и $S$. Наша цель - описать эту группу.

Сделаем предварительно несколько замечаний.

Пользуясь алгоритмом из [12], можно, взяв в качестве примера какую-либо полугруппу $\langle a, b \mid a=b Q a\rangle$, построить копредставления для $S$ и для $G$. При "малой" длине слова $b Q$ в результате упрощений часто оказывается, что $S$ может быть задана одним определяюшим соотношением. Однако этот факт не справедлив в общем случае. Например, если П задана соотношением $a=b a^{2} b a b^{2} a^{2}$, то $S$ имеет задание с помошью трех порождаюших $x, y, z$ и двух соотношений: $x=y z x z$, $y=z y z$. Можно доказать, что $S$ не может быть задана в этом случае одним определяюшим соотношением (ни для какого множества порождающих).

Однако оказывается, что группа $G$ в любом случае задается одним определяюшим соотношением, что мы и докажем ниже. Поскольку в $G$ проблема слов разрешима в силу классического результата Магнуса [22] (см. также [9]), то она бу- 
дет разрешима и в $S$. Значит, мы умеем решать проблему слов в подполугруппе $S$ полугруппы П. Чтобы распространить это на всю полугруппу П, было бы достаточно решить проблему левой делимости для $S$ (ввиду весьма общего результата статьи [12]).

Отметим без доказательства, что достаточно решить также проблему вхождения в $S$ внутри П (т.е. представить алгоритм, который по данному слову от $a, b$ определял бы, будет ли оно равно в П какому-либо слову из $S$ ).

Далее мы будем существенно опираться на результаты, полученные в [11], [12]. Для этого нам потребуется напомнить наиболее важные из них, а также кое-какие определения.

В [12] рассматриваются полугруппы, заданные определяюшими соотношениями без левых циклов. Для таких полугрупп разрешимость проблемы равенства вытекает из разрешимости проблемы левой делимости (см., например, [4]). Пусть П - полугруппа без левых циклов. В [12] ей сопоставляется полугруппа, обозначаемая $S(\Pi)$. Там же доказывается (теорема 1), что проблема левой делимости в П сводится к проблеме левой делимости в $S(\Pi)$. Полугруппа $S(\Pi)$ описывается с помощью некоторой специальной конструкции, приведенной в [4]. Вообще говоря, не известен алгоритм, который по копредставлению для П позволял бы строить копредставление для $S(\Pi)$. Однако для полугрупп $\langle a, b \mid a=b Q a\rangle$ такое эффективное построение возможно, и оно описано в [12]. Нам понадобится это построение, и мы воспроизведем его в настояшей работе, но для начала сделаем одно замечание.

Из [12] нетрудно извлечь, что на самом деле $S(\Pi)$ есть (с точностью до изоморфизма) подполугруппа в П, порожденная всеми собственными непустыми концами определяющих слов для полугруппы П. Мы будем называть ее подполугруппой концов. Отсюда, в частности, следует, что утверждение, обратное упоминавшейся теореме 1 , также верно (при этом нужно учесть [12, лемма 5]). Таким образом, проблемы левой делимости для П и $S(\Pi)$ равносильны.

Напомним описание определяюших соотношений для $S(\Pi)$. Пусть $x, y$ - какие-либо собственные непустые конщы определяющих слов, задающих П. Допустим, что $x, y$ сравнимы в П слева. Тогда можно некоторым определенным образом указать слова $f(x, y)$ и $f(y, x)$, каждое из которых есть произведение непустых собственных конщов определяюших полугруппу П слов (в дальнейшем мы просто будем говорить о концах), так что $x f(x, y)=y f(y, x)$ в П.

Напомним определение функции $f(x, y)$ из [12], давая описание в несколько видоизмененной форме. Имея полугруппу П с единицей, заданную копредставлением без левых циклов, определим для каждой пары слов $X, Y$, сравнимых в П слева, слова, обозначаемые $f(X, Y)$ и $f(Y, X)$. Именно, существуют слова $Z, T$ такие, что в П выполнено равенство $X Z=Y T$. При этом имеется диаграмма над исходным копредставлением, осуществляющая равенство слов $X Z$ и $Y T$. Выберем при фиксированных $X$ и $Y$ такую диаграмму, которая имеет как можно меньше клеток, а при фиксированном числе клеток - как можно меньше ребер. В [12] показано (с использованием выводов вместо диаграмм), что при этих условиях слова $Z$ и $T$, соответствующие такой диаграмме, находятся однозначно. По определению $f(X, Y) \equiv Z$ и $f(Y, X) \equiv T$. Очевидно, в П выполнено равенство $X f(X, Y)=Y f(Y, X)$. 
Для получения копредставления полугрупш $S(\Pi)$ нужно сопоставить различным концам различные буквы нового алфавита (это будут порождающие $S(\Pi)$ ) и для каждой пары сравнимых слева в П конщов записать соотношение вида $x f(x, y)=y f(y, x)$, заменяя концы на соответствуюшие буквы. (Неэффективность этого задания связана с тем, что мы априори не знаем, какие конщы сравнимы слева в П, а какие нет.)

Для случая полугрупп вида $\Pi=\langle a, b \mid a=b Q a\rangle$, как уже говорилось, эффективное построение $S(\Pi)$ возможно. Прежде чем изложить соответствующий алгоритм (которьй содержится в [12]), мы напомним один весьма нетривиальньй результат статьи $[11]$ :

если $\Pi=\langle a, b \mid a=b Q a\rangle$, то никакой (собственный) конец слова $b Q a$, начинающийся буквой $b$, не делится на $a$ слева в полугруппе П.

Этот факт мы в дальнейшем будем назьвать леммой Оганесяна.

Обозначим $b Q a \equiv b e_{n} \ldots e_{1}$, где $e_{1}, \ldots, e_{n}$ - буквы. Пусть $Y_{i} \equiv e_{i} \ldots e_{1}(1 \leqslant$ $i \leqslant n)$; буквы $y_{1}, \ldots, y_{n}$ нового алфавита соответствуют словам $Y_{i}$. Ниже будет реализован индуктивньй алгоритм, выясняюший сравнимость слева слов вида $Y_{i}$, $Y_{j}$. Следствие 1.3 позволяет заключить, что сравнимость этих слов слева означает, что одно из них делится слева на другое. Пусть $Y_{i}$ делится слева на $Y_{j}$. При этом оказывается, что $Y_{i}$ равно в $S$ слову вида $Y_{j} Y_{k_{1}} \ldots Y_{k_{r}}$ для некоторых $k_{1}, \ldots$, $k_{r}$. В качестве определяюших соотношений для $S(\Pi)$ будут взяты все выражения вида $y_{i}=y_{j} y_{k_{1}} \ldots y_{k_{r}}$.

Проводя индукцию по $i$ и используя вышеизложенное, мы будем выяснять, какие из слов $Y_{j}$ делятся на $Y_{i}$. Далее будет найдено слово $W_{i j}\left(Y_{1}, \ldots, Y_{n}\right)$ такое, что $Y_{j}=Y_{i} W_{i j}\left(Y_{1}, \ldots, Y_{n}\right)$ в $S$. Тогда $y_{j}=y_{i} W_{i j}\left(y_{1}, \ldots, y_{n}\right)$ будет одним из определяющих соотношений для $S(\Pi)$. По сути мы повторяем здесь в несколько видоизмененной форме доказательство леммы 8 из [12]. Это необходимо сделать, так как описываемый процесс (алгоритм Оганесяна) существенно используется далее.

1) $i=1$. Нужно выяснить, какие из слов $Y_{j}$ делятся в П слева на $a$. Ответ дается леммой Оганесяна: те и только те слова, которые начинаются буквой $a$. Имеем $Y_{j} \equiv Y_{1} Y_{j-1}$, где $j>1, e_{j}=a$. Полагаем $W_{1 j}\left(y_{1}, \ldots, y_{n}\right)=y_{j-1}$ при $j>1, e_{j}=a$.

2) Пусть $i>1$, и пусть мы знаем, какие из конщов делятся на $Y_{i-1}$. Выясним, какие из конщов делятся на $Y_{i}$. При этом определяющие соотношения, наложенные на шагах с номерами, меньшими $i$, уже получены.

Имеем $Y_{j} \equiv e_{j} Y_{j-1}, Y_{i} \equiv e_{i} Y_{i-1}$. Если $e_{j}=e_{i}$, то $Y_{j}$ делится на $Y_{i}$ слева тогда и только тогда, когда $Y_{j-1}$ делится на $Y_{i-1}$ слева (ввиду того, что в П, рассматриваемой вместе с единицей, выполнен закон левостороннего сокращения). Если делимость имеет место (при $j \neq i$ ), то слово $Y_{j-1}$ непусто, откуда $j>1$. Мы имеем в П равенство $Y_{j-1}=Y_{i-1} W_{i-1, j-1}$. Следовательно, равенство $Y_{j}=Y_{i} W_{i-1, j-1}$ также имеет место в $\Pi$, и можно положить $W_{i j}=W_{i-1, j-1}$, добавляя соотношение $y_{j}=y_{i} W_{i j}\left(y_{1}, \ldots, y_{n}\right)$.

Пусть $e_{i} \neq e_{j}$. Если $e_{i}=a, e_{j}=b$, то $Y_{j}$ не делится на $Y_{i}$ слева, так как оно не делится слева на $a$ ввиду леммы Оганесяна. Рассмотрим случай $e_{i}=b, e_{j}=a$. Предположим, что $Y_{j} \equiv a Y_{j-1}$ делится слева на $Y_{i} \equiv b Y_{i-1}$. Но тогда слова $a$ и $b Y_{i-1}$ сравнимы слева в П. Ввиду следствия 1.3 одно из них делится слева на другое. Поэтому мы имеем, что $a$ делится слева на $b Y_{i-1}$ ввиду леммы Оганесяна. 
Поэтому $Y_{j}$ делится на $Y_{i}$ слева тогда и только тогда, когда $a$ делится на $Y_{i}$ слева, так как $Y_{j}$ начинается буквой $a$. Так как $a=b Q a=b Y_{n}$ и в П выполнен закон левостороннего сокращения, то данный факт означает, что $Y_{n}$ делится слева на $Y_{i-1}$ в П. Последний факт мы умеем распознавать. Если это имеет место, то определено слово $W_{i-1, n}\left(Y_{1}, \ldots, Y_{n}\right)$, и в П верно равенство $Y_{n}=Y_{i-1} W_{i-1, n}$. Тогда $Y_{j}=a Y_{j-1}=b Y_{n} Y_{j-1}=b Y_{i-1} W_{i-1, n} Y_{j-1}=Y_{i} W_{i-1, n} Y_{j-1}$, и можно взять в качестве $W_{i, j}$ слово $W_{i-1, n} Y_{j-1}$, добавляя соотношение $y_{j}=y_{i} W_{i j}\left(y_{1}, \ldots, y_{n}\right)$.

На этом построение $S(\Pi)$ закончено; этот процесс эффективен. Таким образом,

$$
\left.S(\Pi)=\left\langle y_{1}, \ldots, y_{n}\right| y_{j}=y_{i} W_{i j} \quad\left(i \neq j, Y_{j} \text { делится на } Y_{i} \text { слева }\right)\right\rangle .
$$

Одним из основных (и весьма глубоких) результатов работы [12] является теорема 2 , утверждаюшая, что $S(\Pi)$ можно задать системой определяюших соотношений как без левых, так и без правых циклов. (Копредставление (4), вообше говоря, может иметь циклы, однако оно может быть упрощено и приведено к нужному виду.)

Любая полугруппа, заданная соотношениями без циклов, может быть изоморффно вложена в группу [3].

Из вполне элементарных соображений следует, что если полугруппа $S$ задана некоторым копредставлением и при этом вложима в некоторую группу, то она вложима в группу $G$, заданную этим же копредставлением.

Возникает вполне резонный вопрос: какова эта группа для случая полугруппы $S(\Pi)$ ? Ответом на него является

TEорема 2.1. Полугруппа $S(\Pi)$, порожденная в полугруппе П вида $\langle a, b|$ $a=b Q a\rangle$ всеми непустыми концами определяющего слова $b Q a$, изоморфно влохиим в некоторую группу $G(\Pi)$ с одним определяющим соотношением. При әтом соотношение, задающее группу $G(\Pi)$, а также вложение $S(\Pi)$ в $G(\Pi)$ находятся эффективно по данному слову $b Q a$.

(В процессе доказательства будет уточнено, как именно выглядит соотношение, определяющее $G(\Pi)$. Заметим сразу, что оно не всегда имеет вид $b Q=1$.)

ДокАЗАТЕльство. Так как $S(\Pi)$ вкладывается в группу, она вкладывается в группу

$$
G(\Pi)=\left\langle y_{1}, \ldots, y_{n} \mid y_{j}=y_{i} W_{i j}\left(y_{1}, \ldots, y_{n}\right)\right\rangle,
$$

заданную групповым копредставлением (соотношения взяты для тех случаев, когда слова $W_{i j}$ определены, т.е. при $i \neq j$ и при условии, что $Y_{j}$ делится на $Y_{i}$ слева в П).

Мы докажем, что $G(\Pi)$ изоморфна группе, заданной одним определяющим соотношением, которая будет обозначена $\bar{G}(\Pi)$. Перейдем к описанию последней.

Построим неориентированньй граф с вершинами $y_{1}, \ldots, y_{n}$. Если $Y_{i}$ делится слева на $Y_{j}$ в П (при $i \neq j$ ), то соединим $y_{i}$ с $y_{j}$ при помоши ребра. Данный графф строится эффективно ввиду наличия алгоритма, выясняюшего сравнимость (делимость) слева для концов. Полученный граф разбивается на связные компоненты. Компонента, которой принадлежит вершина $y_{1}$ (назовем ее главной), содержит все такие вершины $y_{i}$, для которых $Y_{i}$ начинается буквой $a$, а также такие вершины 
$y_{j}$, для которых $Y_{j}$ начинается буквой $b$, но при этом $a$ делится слева на $Y_{j}$. Множество всех остальных связных компонент графа обозначим через $\Omega$ (оно может быть пустым). Введем новые буквы $b_{\omega}$ для всех $\omega \in \Omega$. Положим $\bar{e}_{i}=e_{i}$, если $y_{i}$ принадлежит главной связной компоненте графа. Пусть $y_{i}$ принадлежит компоненте $\omega$. Тогда $e_{i}=b$. В этом случае положим $\bar{e}_{i}=b_{\omega}$. Рассмотрим равенство вида $b \bar{e}_{n} \ldots \bar{e}_{1}=a$ (каждое $\bar{e}_{i}$ есть либо $a$, либо $b$, либо одно из $\left.b_{\omega}\right)$. Оно и будет взято в качестве соотношения, определяющего $\bar{G}(\Pi)$. Ясно, что $\bar{e}_{1}=a$, и это равенство можно сразу сократить справа на $a$. Таким образом,

$$
\bar{G}(\Pi)=\left\langle a, b, b_{\omega}(\omega \in \Omega) \mid b \bar{e}_{n} \ldots \bar{e}_{2}=1\right\rangle .
$$

Эта группа строится эффективно по копредставлению П. Нам осталось доказать, что она изоморфна $G(\Pi)$.

Сейчас мы прервем доказательство, чтобы проиллюстрировать построение $\bar{G}(\Pi)$ на двух примерах.

ПРИмеР 2.1. $\Pi=\langle a, b \mid a=b b a b a\rangle$.

Выпишем соотношения, определяюшие $S(\Pi)$, пользуясь изложенным вьше алгоритмом Оганесяна. Для краткости будем временно обозначать делимость слева слова $X$ на слово $Y$ в П при помощи записи $X \succeq Y$.

При $i=1$ мы имеем, что лишь $Y_{j}=Y_{3}$ делится на $Y_{1}=a$; полагаем $y_{3}=y_{1} y_{2}$. При $i=2$ мы видим, что $a=Y_{1}$ не делится слева на $Y_{2}$, так как $a \succeq Y_{2} \Longleftrightarrow b Y_{4} \succeq$ $b Y_{1} \Longleftrightarrow Y_{4} \succeq Y_{1}=a$, но последнее не имеет места. Отсюда следует, что $Y_{3}$ не делится слева на $Y_{2}$, так как начинается с $a$, и это равносильно условию делимости $a$ на $Y_{2}$. Далее, $Y_{4} \succeq Y_{2} \Longleftrightarrow b Y_{3} \succeq b Y_{1} \Longleftrightarrow Y_{3} \succeq Y_{1}$; мы получаем, что $y_{4}=y_{2}^{2}$. При $i=3$ очевидно, что никакой другой из конщов не делится на $Y_{3}$. Аналогичный факт верен и для $i=4$. (Вообще, если для некоторого $i$ условие $Y_{j} \succeq Y_{i}$ влечет $j=i$, то и для всех $i<k \leqslant n$ верно, что $Y_{j} \succeq Y_{k}$ влечет $j=k$, что нетрудно вывести из описанного алгоритма.)

Таким образом, $S(\Pi)=\left\langle y_{1}, y_{2}, y_{3}, y_{4} \mid y_{3}=y_{1} y_{2}, y_{4}=y_{2}^{2}\right\rangle$, т.е. $S(\Pi)-$ свободная полугруппа с двумя порождающими. При этом, конечно же, группа $G(\Pi)$, заданная тем же копредставлением, есть свободная группа с двумя порождающими $y_{1}, y_{2}$. Нас интересует группа $\bar{G}(\Pi)$. Рассмотрим граф, изображенный на рис. 1 . Он имеет одну не главную компоненту. Свяжем с ней новую букву $c$. Тогда определяющее соотношение для $\bar{G}(\Pi)$ получится заменой букв $e_{2}, e_{4}$, соответствующих этой компоненте, на букву $c$. Имеем для $\bar{G}(\Pi)$ копредставление $\langle a, b, c \mid b c a c=1\rangle$. Ясно, что получилась свободная группа $\langle a, c\rangle$ ранга 2.

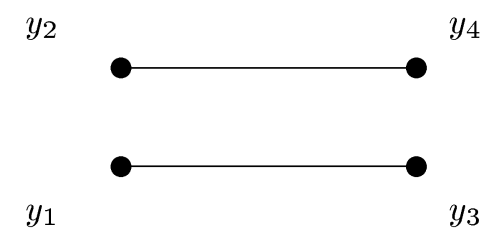

Рис. 1 
ПРИмеР 2.2. $\Pi=\langle a, b \mid a=b a a b a\rangle$.

Не будем прослеживать ход алгоритма Оганесяна в деталях; выпишем последовательно получаюшиеся при этом соотношения для $S(\Pi)$. Имеем $y_{3}=y_{1} y_{2}$, $y_{4}=y_{1} y_{3}, y_{1}=y_{2} y_{3}, y_{3}=y_{2} y_{3} y_{2}, y_{4}=y_{2} y_{3}^{2}, y_{4}=y_{3}^{2} y_{2}$. Занумеруем эти соотношения так: $(1),(2), \ldots,(6)$. Понятно, что (5) следует из (2) и (3), а (6) - из (1), (4) и (2). Удаляя (5) и (6), видим, что можно удалить $y_{4}$ вместе с (2). Так как (1) следует из (3) и (4), можно удалить (1), а затем удалить $y_{1}$ вместе с (3). Получаем, что $S(\Pi)=\left\langle y_{2}, y_{3} \mid y_{3}=y_{2} y_{3} y_{2}\right\rangle$.

Очевидно, что граф будет иметь только главную компоненту, поэтому соотношение для $\bar{G}(\Pi)$ получается просто сокрашением на $a$.

Имеем: $\bar{G}(\Pi)=\langle a, b \mid b a a b=1\rangle \cong\left\langle a, b \mid b^{2} a^{2}=1\right\rangle$ - свободное произведение с объединенной подгруппй. (Можно непосредственно видеть, что группа $G(\Pi)=$ $\left\langle y_{2}, y_{3} \mid y_{3}=y_{2} y_{3} y_{2}\right\rangle$ изоморфна данной.)

Продолжим доказательство теоремы. Наша цель - построить взаимно обратные гомоморфизмы $G(\Pi)$ и $\bar{G}(\Pi)$ друг на друга.

Сопоставим букве $y_{i}$ слово $\bar{e}_{i} \ldots \bar{e}_{1}$. Таким образом, мы имеем гомоморфизм $\varphi$ свободной группы $\left\langle y_{1}, \ldots, y_{n}\right\rangle$ в $\bar{G}(\Pi)$, заданный с помощью правила $\varphi\left(y_{i}\right)=$ $\bar{e}_{i} \ldots \bar{e}_{1}$. Проверим, что под действием этого правила все определяюшие соотношения группы $G(\Pi)$ переходят в равенства, выполненные в $\bar{G}(\Pi)$; тогда будет определен индуцированный гомоморфизм $G(\Pi)$ в $\bar{G}(\Pi)$, который далее также будет обозначаться через $\varphi$. Будем проверять этот факт, проводя индукцию по $i$, в поцессе появления соотношений вида $y_{j}=y_{i} W_{i j}$.

При $i=1$ равенства вида $y_{i}=y_{1} y_{i-1}$, где $e_{i}=a$, перейдут в равенства $\bar{e}_{i} \ldots \bar{e}_{1}=\bar{e}_{1} \bar{e}_{i-1} \ldots \bar{e}_{1}$, которые справедливы, так как $\bar{e}_{i}=\bar{e}_{1}=a$.

Пусть $i>1$ и данное свойство выполняется для $i-1$. Допустим, что мы имеем соотношение $y_{j}=y_{i} W_{i j}$, где $e_{j}=e_{i}$. Заметим, что $W_{i-1, j-1}=W_{i j}$, и мы имеем соотношение $y_{j-1}=y_{j-1} W_{i-1, j-1}$, которое переходит в верное равенство под действием $\varphi$. Так как $\bar{e}_{j}=\bar{e}_{i}$, образ нужного нам равенства в $\bar{G}(\Pi)$ отличается от предыдушего домножением слева на одну и ту же букву, т.е. оно также верно.

Пусть $e_{j}=a, e_{i}=b$ и имеется соотношение $y_{j}=y_{i} W_{i j}$. Это значит, что на предыдущем шаге мы имели $y_{n}=y_{i-1} W_{i-1, n}$, a $W_{i j}=W_{i-1, n} y_{j-1}$. Отсюда получаем, что $\varphi\left(y_{n}\right)=\varphi\left(y_{i-1} W_{i-1, n}\right)$ в $\bar{G}(\Pi)$. Нужно убедиться, что $\varphi\left(y_{j}\right)=\varphi\left(y_{i} W_{i j}\right)$ в $\bar{G}(\Pi)$. Заметим, что $Y_{j}$ делится на $Y_{i}$, а потому $y_{i}$ принадлежит главной компоненте графа. Следовательно, $\bar{e}_{i}=b$. Тогда в $\bar{G}(\Pi)$ имеем (поскольку $b \bar{e}_{n} \ldots \bar{e}_{1}=a$ есть соотношение в $\bar{G}(\Pi))$

$$
\begin{aligned}
\varphi\left(y_{j}\right) & =\bar{e}_{j} \bar{e}_{j-1} \ldots \bar{e}_{1}=a \varphi\left(y_{j-1}\right)=b \bar{e}_{n} \ldots \bar{e}_{1} \varphi\left(y_{j-1}\right)=b \varphi\left(y_{n}\right) \varphi\left(y_{j-1}\right) \\
& =b \varphi\left(y_{i-1}\right) \varphi\left(W_{i-1, n}\right) \varphi\left(y_{j-1}\right)=\bar{e}_{i} \ldots \bar{e}_{1} \varphi\left(W_{i-1, n} y_{j-1}\right) \\
& =\varphi\left(y_{i}\right) \varphi\left(W_{i j}\right)=\varphi\left(y_{i} W_{i j}\right),
\end{aligned}
$$

что и требовалось доказать.

Мы определили гомоморфизм $\varphi: G(\Pi) \rightarrow \bar{G}(\Pi)$. Для того чтобы определить гомоморфизм $\psi: \bar{G}(\Pi) \rightarrow G(\Pi)$, нам потребуется один вспомогательный факт.

Пусть $\bar{e}_{i}=\bar{e}_{j}$. Докажем, что $y_{i} y_{i-1}^{-1}=y_{j} y_{j-1}^{-1}$ в $G(\Pi)$. (При этом считаем по определению, что $y_{0}=1$.) Ясно, что $y_{i}, y_{j}$ принадлежат одной и той же компоненте графа. Очевидно также, что $e_{i}=e_{j}$. Достаточно рассмотреть случай, когда, 
скажем, $Y_{j}$ делится в П слева на $Y_{i}$. K этому сводится обший случай, так как компонента связна, а равенство $y_{i} y_{i-1}^{-1}=y_{j} y_{j-1}^{-1}$ будет установлено для случая, когда $y_{i}, y_{j}$ соединены ребром. Но тогда оно будет верно для любых вершин компоненты. Итак, считаем, что $Y_{j}$ делится слева на $Y_{i}, j \neq i$. Если $i=1$, то $y_{j}=y_{1} y_{j-1}$ в П, т.е. равенство верно. Пусть $i>1$. Тогда на $i$-м шаге возникает соотношение вида $y_{j}=y_{i} W_{i j}\left(y_{1}, \ldots, y_{n}\right)$. Поскольку $e_{j}=e_{i}$, это соотношение могло возникнуть только как следствие соотношения $y_{j-1}=y_{i-1} W_{i-1, j-1}\left(y_{1}, \ldots, y_{n}\right)$, причем $W_{i j}=W_{i-1, j-1}=W$. Отсюда вытекает то, что нам нужно:

$$
y_{j} y_{j-1}^{-1}=y_{i} W\left(y_{i-1} W\right)^{-1}=y_{i} y_{i-1}^{-1} .
$$

Теперь зададим гомоморфизм $\left\langle a, b, b_{\omega}(\omega \in \Omega)\right\rangle \longrightarrow G(\Pi)$, определяя образы букв. Положим $a \mapsto y_{1}, b \mapsto y_{1} y_{n}^{-1}, b_{\omega} \mapsto y_{i} y_{i-1}^{-1}$, где $y_{i} \in \omega$ (ввиду доказанного выше образ $b_{\omega}$ не зависит от представителя $y_{i}$ компоненты $\omega$ ). Мы утверждаем, что $\bar{e}_{i} \mapsto y_{i} y_{i-1}^{-1}$.

Этот факт нуждается в доказательстве лишь для случая $\bar{e}_{i}=b$. Если это так, то $e_{i}=b$, и $y_{i}$ принадлежит главной компоненте. Допустим вначале, что $a$ делится слева на $Y_{i}$. Тогда на $i$-м шаге (заметим, что $i>1$ ввиду $e_{i}=b$ ) появляется соотношение $y_{1}=y_{i} W_{i 1}$, которое получается из соотношения $y_{n}=y_{i-1} W_{i-1, n}$, имевшего место на $(i-1)$-м шаге, причем $W_{i 1}=W_{i-1, n}=W$ (вспомним, что $W_{i j}$ в этом случае есть $\left.W_{i-1, n} Y_{j-1}=W_{i-1, n} Y_{0}=W_{i-1, n}\right)$. Но теперь $y_{1} y_{n}^{-1}=$ $y_{i} W\left(y_{i-1} W\right)^{-1}=y_{i} y_{i-1}^{-1}$, что мы и хотели получить. Таким образом, $y_{i} y_{i-1}^{-1}=$ $y_{1} y_{n}^{-1}$ для вершин $y_{i}$ из главной компоненты с условием $e_{i}=b$, если $a$ делится слева на $Y_{i}$ (т.е. если $y_{1}=a$ соединено с $y_{i}$ ребром).

Возьмем теперь любую вершину $y_{i}$ такую, что $\bar{e}_{i}=b$. Поскольку главная компонента связна и состоит не только из $a$, должно найтись такое $j$, что $y_{j}$ соединено ребром с $a$. Только что было установлено, что $y_{j} y_{j-1}^{-1}=y_{1} y_{n}^{-1}$. Учитывая, что $\bar{e}_{i}=\bar{e}_{j}$, согласно доказанному вьше вспомогательному факту имеем равенство $y_{i} y_{i-1}^{-1}=y_{j} y_{j-1}^{-1}$ в $G(\Pi)$. В итоге имеем $y_{i} y_{i-1}^{-1}=y_{1} y_{n}^{-1}$, что и требовалось проверить.

Теперь, зная, что $\bar{e}_{i} \mapsto y_{i} y_{i-1}^{-1}$, мы получаем

$$
b \bar{e}_{n} \ldots \bar{e}_{2} \mapsto y_{1} y_{n}^{-1} y_{n} y_{n-1}^{-1} \ldots y_{2} y_{1}^{-1}=1
$$

в $G(\Pi)$, а это и означает наличие гомоморфизма $\psi: \bar{G}(\Pi) \rightarrow G(\Pi)$ такого, что $\psi(a)=y_{1}, \psi(b)=y_{1} y_{n}^{-1}, \psi\left(b_{\omega}\right)=y_{i} y_{i-1}^{-1}\left(y_{i}-\right.$ представитель компоненты $\left.\omega \in \Omega\right)$.

Осталось проверить равенства $\psi \varphi\left(y_{i}\right)=y_{i}$ в $G(\Pi), \varphi \psi(a)=a, \varphi \psi(b)=b$, $\varphi \psi\left(b_{\omega}\right)=b_{\omega}(\omega \in \Omega)$ в $\bar{G}(\Pi)$. Имеем

$$
\begin{aligned}
\psi \varphi\left(y_{i}\right) & =\psi\left(\bar{e}_{i} \ldots \bar{e}_{1}\right)=\psi\left(\bar{e}_{i}\right) \ldots \psi\left(\bar{e}_{1}\right)=y_{i} y_{i-1}^{-1} y_{i-1} y_{i-2}^{-1} \ldots y_{2} y_{1}^{-1} y_{1}=y_{i}, \\
\varphi \psi(a) & =\varphi\left(y_{1}\right)=\bar{e}_{1}=a, \\
\varphi \psi(b) & =\varphi\left(y_{1} y_{n}^{-1}\right)=a\left(\bar{e}_{n} \ldots \bar{e}_{2} \bar{e}_{1}\right)^{-1}=\left(\bar{e}_{n} \ldots \bar{e}_{2}\right)^{-1}=b
\end{aligned}
$$

(так как $b \bar{e}_{n} \ldots \bar{e}_{2}=1$ есть определяюшее соотношение для $\bar{G}(\Pi)$ ); если $y_{i}-$ представитель компоненты $\omega$, то

$$
\varphi \psi\left(b_{\omega}\right)=\varphi\left(y_{i} y_{i-1}^{-1}\right)=\bar{e}_{i} \ldots \bar{e}_{1} \bar{e}_{1}^{-1} \ldots \bar{e}_{i-1}^{-1}=\bar{e}_{i}=b_{\omega} .
$$

Теорема полностью доказана. (Вложение $S(\Pi)$ в $\bar{G}(\Pi)$ эффективно, так как мы умеем вычислять образ $y_{i}$ в $\bar{G}(\Pi)$.)

Теперь можно сформулировать основные следствия данного результата. 
СлЕДСТВИЕ 2.1. Проблема левой делимости для полугруппь П вида $\langle a, b|$ $a=b Q a\rangle$ сводится к проблеме вхождения әлементов группь $\bar{G}(\Pi)$ с одним определяющим соотношением в конечно порожденную подполугруппу (последняя порождается әлементами $\bar{e}_{i} \ldots \bar{e}_{1} \in G(\Pi)$, где $\left.1 \leqslant i \leqslant n\right)$.

ДокАЗАТЕльСтво. В силу [12, теорема 1] проблема левой делимости для П сводится к аналогичной проблеме для $S(\Pi)$. Пусть $u, v$ - слова от $y_{1}, \ldots, y_{n}$, представляющие элементы $S(\Pi)$. Ясно, что $u$ делится на $v$ слева тогда и только тогда, когда сушествует слово $w\left(y_{1}, \ldots, y_{n}\right)$ (возможно, пустое), для которого $u=v w$ в $S(\Pi)$. Это равносильно сушествованию (позитивного) слова $w$ от $y_{1}, \ldots, y_{n}$, для которого $u=v w$ в $G(\Pi)$ в силу вложимости $S(\Pi)$ в $G(\Pi)$. Но последнее означает, что $v^{-1} u$ принадлежит в $G(\Pi)$ подполугруппе с единицей, порожденной элементами $y_{1}, \ldots, y_{n}$. Однако свойство слова быть равным единице в группе с одним соотношением можно распознать эффективно по теореме Магнуса [22], поэтому речь может идти просто о подполугруппе. Осталось вспомнить, что $G($ П) изоморфна $\bar{G}(\Pi)$, и $y_{i}$ переходит в $\bar{e}_{i} \ldots \bar{e}_{1}$ при этом изоморфизме. Следствие доказано.

СлеДСТВИЕ 2.2. В подполугруппе концов для полугруппь вида $\langle a, b| a=$ bQa〉 разрешима проблема равенства слов.

ДокАЗАТЕЛЬСТво. Подполугруппа конщов $S(\Pi)$ для полугруппы П $=\langle a, b|$ $a=b Q a\rangle$ вкладывается в группу с одним определяюшим соотношением по теореме 2.1. Согласно теореме Магнуса [22] в такой группе разрешима проблема слов, что влечет ее разрешимость для $S(\Pi)$. Следствие доказано.

Нетрудно понять, что проблема вхождения в конечно порожденную подполугруппу в группе с одним соотношением выглядит намного сложнее, чем проблема равенства слов. $\mathrm{K}$ тому же, если мы даже имеем проблему вхождения в подполугруппу, заданную самими порождающими группы, то и этот вопрос остается открытым. Он представляет интерес для решения проблем делимости в полугруппах, заданных одним несократимым слева и справа определяюшим соотношением. Последняя проблема открыта (с учетом ошибочности работы [17]), хотя проблема равенства слов для этого случая решена сравнительно давно [2], [3].

Помимо этого можно показать, что в качестве соотношения для групшы $\bar{G}(\Pi)$ может появиться любое позитивное слово от $a, b$. Хотя такие группы и не составляют класс всех групп с одним определяющим соотношением (скажем, известно, что все такие группы аппроксимируются разрешимыми группами [18], что неверно для групп с одним соотношением в целом), но все же думается, что это не очень сильное ограничение.

Поэтому, на наш взгляд, проблема левой делимости для полугрупп вида $\Pi=$ $\langle a, b \mid a=b Q a\rangle$ по существу равносильна проблеме вхождения, о которой шла речь. По-видимому, это весьма сложная проблема. Следует заметить, что метод Магнуса, позволяюший решить проблему равенства слов в группах с одним соотношением, вряд ли применим к проблеме вхождения. Он связан с техникой $\mathrm{HNN}$-расширений, однако группа $F_{2} \times F_{2}$, имеюшая неразрешимую проблему вхождения в некоторые свои конечно порожденные подгрупшы [10], легко представима в виде последовательных HNN-расширений группы $\mathbf{Z}$.

Априори можно было бы ожидать, что проблема равенства слов для П все-таки проще, чем проблема левой делимости. Вполне естественно выглядела бы гипоте- 
за, что проблема слов для П могла бы быть каким-то образом сведена к проблемам типа проблемы равенства слов в группах с одним соотношением. Однако такие попытки, как нам представляется, вряд ли могут привести к успеху.

Дело в том, что ниже будет доказано, что проблема равенства слов для П равносильна проблеме левой делимости для П, хотя на первый взгляд она и выглядит проще.

Однако прежде чем сделать это, мы установим ряд положительных результатов для некоторых частных случаев. Известно, что проблема вхождения в конечно порожденную подполугруппу разрешима в свободной группе [19]. Отсюда выводим

СлЕДСТВИЕ 2.3. Если $\Pi=\langle a, b \mid a=b Q a\rangle$ u әруnпа $G(\Pi)$ свободна, то в $\Pi$ разрешимы проблема равенства слов и проблема левой делимости.

Заметим, что по данной группе с одним определяющим соотношением можно эффективно определить, будет ли она свободной [9, предложения 5.10 и 2.26$]$. Тем самым проверка посылки следствия 2.3 может быть осуществлена эффективно. Мы можем попытаться найти более слабые, но зато легче проверяемые достаточные условия для того, чтобы группа $G(\Pi)$ была свободной.

Г.У. Оганесян доказал в [11], что если $b Q$ есть истинная степень, то проблема левой делимости (а значит, и проблема равенства) разрешима в П. Мы к тому же проверим, что $G(\Pi)$ свободна в этом случае, и получим обобщение этого результата. Дадим вначале несколько определений.

Пусть задана полугруппа $\Pi=\langle a, b \mid a=b Q a\rangle$. Назовем конец $Y_{i}$ слова $b Q a$ myпuковылм, если на него не делится слева никакой другой конец (напомним, что $n=|b Q|$, а $Y_{i}$ есть конец слова $b Q a$ длины $i$, где $\left.1 \leqslant i \leqslant n\right)$. Конец $Y_{i}$ назовем изолированным, если он не сравним слева ни с каким другим концом. В силу следствия 1.3 это значит, что он является тупиковым и при этом не делится слева ни на какой другой конец.

Следуя [11], введем лексикографический порядок $\prec$ на множестве всех слов от $a, b$, при котором $b \prec a$. Это значит, что для любых слов $X, Y$ мы имеем $X \prec Y$ тогда и только тогда, когда либо $X$ есть собственное начало $Y$, либо $X \equiv Z b X^{\prime}$, $Y \equiv Z a Y^{\prime}$ для некоторых $Z, X^{\prime}, Y^{\prime}$. Среди всех конщов (т.е. собственных непустых концов слова $b Q a)$ выделим самый младший конец $L$ и самый старший конец $M$.

В работе [11] рассматривается условие $(\beta)$, достаточное для разрешимости в П проблем равенства и левой делимости. Будучи сформулированньм несколько иначе, оно означает, что никакой конец слова $b Q a$, более длинный, чем $L$, не делится слева на $L$ (сюда включается случай самого слова $b Q a$, т.е. $a$ не делится на $L$ слева). Мы покажем, что из выполнения этого условия также следует, что группа $G(\Pi)$ свободна, усиливая тем самым результат из [11]. Рассмотрим следующие условия:

(1) слово $b Q$ есть истинная степень, т.е. $b Q \equiv(b P)^{m}$ при $m>1$;

$(2)$ самый младший в смысле порядка $\prec$ конец $L$ имеет бо́льшую длину, чем самый старший $M$;

(3) выполнено условие $(\beta)$ работы [11], т.е. на $L$ не делится слева никакой более длинный из концов, включая $b Q a$;

(4) имеется хотя бы один изолированный конец;

(5) группа $G(\Pi)$ свободна;

(6) проблемы равенства и левой делимости слов в П разрешимы. 
Tеорема 2.2. Имеют место импликаиии (1) $\Longrightarrow(2) \Longrightarrow(3) \Longrightarrow(4) \Longrightarrow$ $(5) \Longrightarrow(6)$.

Для сравнения заметим, что в [11] доказано $(1) \Longrightarrow(3) \Longrightarrow(6)$.

ДокАЗАТЕЛЬСТво. (1) $\Longrightarrow(2)$. Пусть П задана соотношением $a=(b P)^{m} a$, $m>1$. Проверим, что $M$ есть конец слова $P a$. Если это не так, то $M=M^{\prime} b P a$ для некоторого слова $M^{\prime}$. Так как $M^{\prime} b P$ есть конец слова $(b P)^{m}$, то $M^{\prime}$ есть конец слова $(b P)^{m-1}$. Поэтому $M^{\prime} a$ есть собственный непустой конец определяющего слова. Очевидно, однако, что $M=M^{\prime} b P a \prec M^{\prime} a$ вопреки определению $M$. Далее, проверим, что $L$ оканчивается словом $(b P)^{m-1} a$. Если это не так, то $L=L^{\prime} a$, где $L^{\prime}$ - собственный конец слова $(b P)^{m-1}$. В этом случае $L^{\prime} b P$ - собственньй конец слова $(b P)^{m}$, и потому $L^{\prime} b P a-$ собственный конец определяющего слова. Однако $L^{\prime} b P a \prec L^{\prime} a=L$ вопреки тому, что $L$ самый младший. Так как $m-1 \geqslant 1$, то $L$ должно оканчиваться словом $b P a$, а потому $M$ есть собственный конец $L$, т.е. $L$ длиннее $M$. Это доказывает (2).

$(2) \Longrightarrow(3)$. Докажем вначале одну лемму.

ЛЕмма 2.1. Если $Y_{i}-$ тупиковьй конец, то все конць $Y_{j}$ при $i<j \leqslant n$ также являются тупиковьми.

ДоКАЗАТЕЛЬСТВо. Пусть $Y_{i}$-тупиковый конец. Достаточно показать, что при $i<n$ конец $Y_{i+1}$ также тупиковый. Предположим, что это не так, и пусть $Y_{k}$ делится слева на $Y_{i+1}$ при $k \neq i+1$. Если $e_{k}=e_{i+1}$, то $k>1$ и $Y_{k-1}$ делится на $Y_{i}$ слева вопреки условию. Поэтому $e_{k}=a, e_{i+1}=b$ по лемме Оганесяна. Поскольку тем самым $a$ сравнимо слева с $Y_{i+1}$, то одно из слов делится на другое по следствию 1.3. Вновь применяя лемму Оганесяна, делаем вывод, что $a$ делится слева на $Y_{i+1} \equiv b Y_{i}$. Сокрашая на $b$ слева, получаем, что $Y_{n} \equiv Q a$ делится слева на $Y_{i}$, что опять противоречит условию. Лемма доказана.

Пусть теперь выполнено (2). Если $Y_{i}$ делится на $Y_{j}$ слева при $i \neq j$, то из леммы Оганесяна имеем $Y_{j} \prec Y_{i}$. В частности, $M$ - тупиковый конец. Так как $|L|>|M|$, то по лемме 2.1 конец $L$ также тупиковый. Так как $L \not \equiv a$, то $a=b Q a$ не делится на $L$ слева. Никакой другой конец на $L$ также не делится. Это доказывает (3).

$(3) \Longrightarrow(4)$. Пусть выполнено (3). Проверим, что $L$ изолирован. Поскольку $L-$ самый младший конец, он не делится слева ни на какой другой конец в силу леммы Оганесяна. Покажем, что $L$ является тупиковым. Предположим противное. Рассмотрим среди концов, отличных от $L$, такой, который делится на $L$ слева. При этом будем считать, что выбранный конец $Y_{i}$ с этим свойством максимален по отношению к $\prec$. По условию (3) имеем $|L|>\left|Y_{i}\right|$. Конец $Y_{i}$ не может быть тупиковым, так как иначе тупиковым оказался бы и $L$ ввиду леммы 2.1. Следовательно, при некотором $j \neq i$ конец $Y_{j}$ делится слева на $Y_{i}$. Но тогда $L \prec Y_{i} \prec Y_{j}$ и $Y_{j}$ делится на $L$. Получено противоречие с выбором $Y_{i}$. Таким образом, $L$ - изолированньй конец.

$(4) \Longrightarrow(5)$. Пусть имеется изолированный конец $Y_{i}$. Если $b Q$ не есть степень $b$, то он начинается с $b$. Если же $b Q \equiv b^{m}$, то при $m>1$ изолированным будет конец $L \equiv b^{m-1} a$, а случай $a=b a$ тривиален (группа $G(\Pi)$ будет бесконечной циклической). Таким образом, можно считать, что $Y_{i}$ начинается с $b$. Компонента графа, построенного в доказательстве теоремы 2.1, содержашая $y_{i}$, одноэлементна. Обозначим ее $\omega$. Тогда соотношение $b \bar{e}_{n} \ldots \bar{e}_{2}=1$, задаюшее $G(\Pi)$, содержит 
в точности одно вхождение буквы $b_{\omega}$, и эту букву можно выразить через остальные порождаюшие, удаляя вместе с соотношением. Тем самым имеем в качестве свободного базиса групшы $G(\Pi)$ набор всех порождаюших, кроме $b_{\omega}$.

$(5) \Longrightarrow(6)$. Это в точности следствие 2.3 .

Теорема доказана.

Отметим, что промежуточное условие (2) мы ввели в рассмотрение ввиду его наглядности. Есть еще одно условие, которое очень просто поддается проверке и для которого разрешимость проблем равенства и левой делимости хорошо известна, это условие гиперпростоты ${ }^{1)}$ слова $b Q a$. Легко показать, что для гиперпростого слова $b Q a$ вьполнено и условие (5).

Нетрудно привести примеры, показываюшие, что все рассмотренные выше условия не эквивалентны друг другу.

В заключение этого параграфа отметим, что естественна попытка расширить класс свободных групп на более широкий так, чтобы в этом классе проблема вхождения в конечно порожденную подполугруппу была разрешима. Однако даже во многом близкие по своим свойствам к свободным группам группы с так называемым условием малого сокращения этим свойством не обладают в силу результата Рипса [25].

\section{§ 3. Проблема левой делимости и проблема равенства слов}

Пусть моноид имеет конечное копредставление с непустыми определяющими словами, и пусть в нем выполнен закон левостороннего сокращения. Хорошо известно, что из разрешимости в нем проблемы левой делимости следует разрешимость проблемы равенства. Этот факт часто использовался в применении к полугруппам без левых циклов (см., например, [4]). Для удобства ссылок сформулируем его в виде леммы.

Лемма 3.1. Пусть $\left\langle x_{1}, \ldots, x_{m} \mid A_{i}=B_{i}(1 \leqslant i \leqslant k)\right\rangle$ - копредставление моноида $S$, удовлетворяющего закону левостороннего сокращения, где все определяющие слова непусть. Если в $S$ разрешима проблема левой делимости, то разрешима и проблема равенства слов.

(Для проверки равенства слов $U$ и $V$ достаточно проверить факт делимости слева $U$ на $V$. Затем, найдя некоторое слово $Z$ такое, что $U$ равно в $S$ слову $V Z$, что можно сделать рекурсивным перечислением всех выводов, нужно убедиться в том, что $Z$ пусто.)

Мы хотим показать, что для полугрупп вида $\langle a, b \mid a=b Q a\rangle$ проблема равенства и проблема левой делимости оказываются эквивалентными. (Отметим, что в [14] сформулирован результат о том, что если во всех полугруппах, заданных несократимым слева определяюшим соотношением, разрешима проблема слов, то во всех этих полугруппах разрешима также проблема левой делимости.) Следующая лемма является основной в нашем рассуждении.

\footnotetext{
1) Слово называется әиперпростым, если никакое его собственное непустое начало не является его концом.
} 


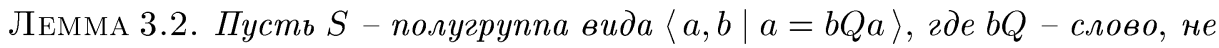
являющееся истинной степенью. Тогда для любого слова $X$ условие, что $X$ делится слева на а в $S$, выполнено тогда и только тогда, когда $b Q X=X$ в $S$.

ДокАЗАТЕльСтво. Часть "только тогда" очевидна: если $X$ делится слева на $a$ в $S$, то $X=a Y$ для некоторого $Y$, и в $S$ выполнены равенства $b Q X=b Q a Y=$ $a Y=X$.

Предположим теперь, что $b Q X=X$ в $S$. Рассмотрим произвольную диаграмму $\Delta$ над копредставлением полугруппы $S$, осушествляюшую равенство слов $b Q X$ и $X$ в $S$. Пусть $G=\langle a, b \mid b Q=1\rangle$. Превратим $\Delta$ в диаграмму $\Delta^{G}$ следующим образом: для каждой клетки диаграммы вида $b Q a=a$ рассмотрим ребро $\mathrm{c}$ “пустой” меткой (рис. 2).
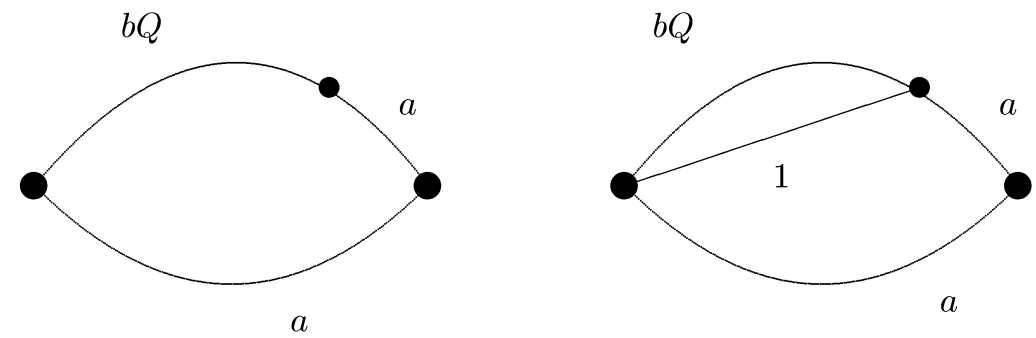

Рис. 2

Говоря точнее, присоединим символ 1 к нашему алфавиту и рассмотрим "тривиальные" соотношения вида $1 \cdot x=x \cdot 1=x(x \in\{a, b\}) ; 1 \cdot 1=1$. Как известно, теория диаграмм надгрупповыми копредставлениями может сталкиваться с рядом топологических трудностей, касаюшихся таких операций, как склейка диаграмм и т.п. В монографиии [15] подробно развивается теория, позволяюшая избежать этих и других трудностей с помощью использования так называемых 0 -клеток (см. [15, $\S 11])$. Следуя этой монографии, мы будем использовать 0-клетки в групповых диаграммах.

Итак, добавляя соответствующие 0-клетки, мы получаем диаграмму $\Delta^{G}$ над копредставлением групшы $G=\langle a, b \mid b Q=1\rangle$ с одним определяющим соотношением.

Теперь рассмотрим диаграмму $\Delta_{0}^{G}$, которая получается из $\Delta^{G}$ следуюшим образом: к верхней границе диаграммы $\Delta_{0}^{G}$, имеющей метку $b Q X$, мы приклеиваем клетку вида $1=b Q$ (рис. 3). Теперь можно склеить верхнюю и нижнюю границы этой диаграммы с использованием 0-клеток, получая сферическую диаграмму.

Однако хорошо известно, что копредставления групп с одним соотношением асферичны $^{2}$. Это означает, что полученная сферическая диаграмма должна быть три-

\footnotetext{
${ }^{2)}$ Имеется целый ряд определений асферичности, которые между собой, вообще говоря, не эквивалентны. Мы используем здесь понятие, которое иногда называют диаграммной асферичностью.
} 


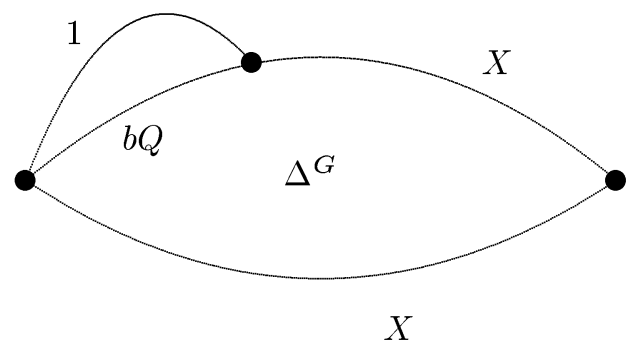

Рис. 3

виальной в некотором смысле. Скажем, что клетки $\pi_{1}$ и $\pi_{2}$ (сферической) диаграммы образуют сократимую пару, если для любой вершины $v_{1}$, принадлежащей контуру $\partial \pi_{1}$ клетки $\pi_{1}$, имеются вершина $v_{2}$, принадлежашая контуру $\partial \pi_{2}$ клетки $\pi_{2}$, и некоторый ${ }^{3)}$ путь $t$ из $v_{1}$ в $v_{2}$ в сферической диаграмме, метка которого равна 1 в свободной группе (рис. 4$)$, причем $\partial \pi_{1}$ и $\partial \pi_{2}$ имеют взаимно обратные метки. Согласно $[9$, предложение 9.7] над копредставлением группы с одним определяюшим соотношением не сушествует приведенных сферических диаграмм. По определению это значит, что можно выделить поддиаграмму из двух клеток, метка граничного контура которой равна единице в свободной группе. Легко показать, что эти клетки образуют сократимую пару.

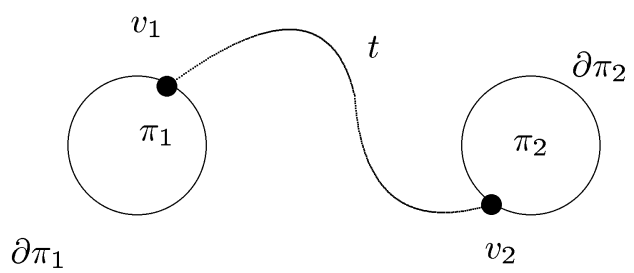

Рис. 4

$\mathrm{B}$ самом деле, метка контура выделенной поддиаграммы имеет вид $R_{1} T R_{2} T^{-1}$, где $R_{1}, R_{2}$ - циклические сдвиги определяюшего слова или обратные к ним слова. Можно считать, что для некоторых слов $X, Y$ выполнены равенства $R_{1} \equiv X Y$, $R_{2} \equiv(Y X) \varepsilon$, где $\varepsilon= \pm 1$. Если $\varepsilon=1$, то имеем равенство $X Y T Y X T^{-1}=1 \mathrm{в}$ свободной группе, из которого следует $X Y T Y X Y=T Y$, т.е. $(X Y T Y)^{2}=(T Y)^{2}$. Это влечет равенство слова $X Y$ единице в свободной группе, что невозможно. Поэтому $\varepsilon=-1$. При этом $X Y$ и $T Y$ коммутируют. Но тогда при некотором $m$ слово $T Y$ равно $(X Y)^{m}$ в свободной группе. В качестве вершин $v_{1}$ и $v_{2}$ возьмем такие точки, чтобы $\partial \pi_{1}$ и $\partial \pi_{2}$ имели метки $X Y$ и $(X Y)^{-1}$ соответственно. Путь $t$ выберем имеюшим метку $T Y(X Y)^{-m}$.

Индукцией по числу клеток докажем, что все клетки нашей сферической диаграммы можно разбить на сократимые пары. Действительно, у нас есть поддиа-

3) Мы не предполагаем, что путь $t$ является несамопересекающимся. 
грамма с меткой контура, равной единище в свободной группе, имеющая две клетки. Удаляя эту поддиаграмму и заклеивая ее 0-клетками, мы получаем сферическую диаграмму с меньшим числом клеток. Применим к ней предположение индукции, разбивая все ее клетки на сократимые пары (если клетки в ней имеются). Прообразы этих клеток в исходной диаграмме также образуют сократимые пары, так как вместо прохождения нового ребра с пустой меткой мы можем проходить по ребрам прежней диаграммы, не меняя значения метки пути в свободной группе. Добавляя к разбиению пару клеток из поддиаграммы, мы имеем искомое разбиение клеток на сократимые пары.

Теперь расклеим сферическую диаграмму, вновь возврашаясь к $\Delta_{0}^{G}$. Клетка $\pi$ имеет парную ей клетку $\pi^{\prime}$. Именно, если $v$-вершина $\pi$, являющаяся концом пути с меткой $b Q$, то имеется некоторая клетка в диаграмме $\Delta_{0}^{G}\left(\right.$ и, конечно, в $\left.\Delta^{G}\right)$ такая, что для некоторой вершины $v^{\prime}$ метка контура $\partial \pi^{\prime}$, читаемого по часовой стрелке с началом и конщом в $v^{\prime}$, равна $b Q$, и к тому же имеется путь $t$ в сферической диаграмме из $v$ в $v^{\prime}$, метка которого равна единице в свободной группе.

Для начала покажем следующее: в $\Delta_{0}^{G}$ (т.е. после расклейки) имеется путь $\bar{t}$, соединяюший $v$ и $v^{\prime}$, метка которого равна единице в свободной группе. Действительно, после расклейки путь $t$ может быть разбит на несколько путей $t_{1}, \ldots, t_{r}$, где конец каждого пути совпадает с началом следующего после склейки. Последнее означает, что эти конец и начало принадлежат соответственно верхнему и нижнему (или наоборот) участку контура диаграммы $\Delta^{G}$, метка каждого из которых равна $X$. Совпадение точек при склейке влечет существование между ними пути (а именно из первой точки в заключительную вершину и далее во вторую точку), метка которого имеет вид $Y Y^{-1}$, а потому равна единице в свободной группе. Следовательно, путь $\bar{t}$ имеется. Но такой путь имеется и в $\Delta^{G}$, так как если мы проходим ребро с меткой 1 , отличающее $\Delta_{0}^{G}$ от $\Delta^{G}$, то мы можем заменить его на путь с меткой $X X^{-1}$. Итак, считаем, что $\bar{t}-$ путь в $\Delta^{G}$.

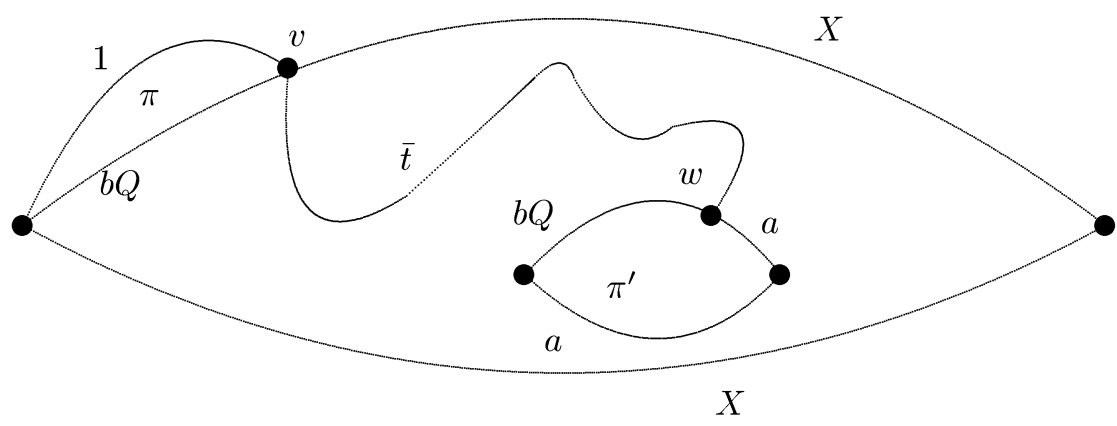

Рис. 5

Ясно, что клетка $\pi^{\prime}$, принадлежащая $\Delta_{0}^{G}$, ориентирована противоположно $\pi$ (рис. 5). Пусть $w$ - вершина на верхней границе контура клетки $\pi^{\prime}$, разбивающая ее на отрезки с метками $b Q$ и $а$ соответственно. Мы утверждаем, что $\bar{t}$ соединяет $v$ и $w$. 
Действительно, если $w$ не совпадает с $v^{\prime}$, то слово, читаемое вдоль контура клетки $\pi^{\prime}$ по часовой стрелке, начиная как с $w$, так и с $v^{\prime}$, есть $b Q$. Но тогда $b Q$ графически равно слову, которое есть его нетривиальный циклический сдвиг. Элементарные факты показывают, что в этом случае $b Q$ есть истинная степень. Поскольку это по условию не так, то $\bar{t}$ соединяет $v$ и $w$.

Теперь воспользуемся следствием 1.2, очевидно, применимым к нашему случаю. В обозначениях следствия 1.2 , имея путь с меткой, равной 1 в свободной группе, мы получаем, что $r(v)=r(w)$. Это означает, что метка любого позитивного пути в $\Delta$ из $v$ в заключительную вершину $\omega$ равна в $S$ метке любого пути из $w$ в заключительную вершину. Однако $w$ есть вершина, выбранная в $\Delta$ как конец некоторого пути с меткой $b Q$ и начало пути с меткой $a$. Следовательно, слово $X$ (идущее из $v$ в $\omega$ ) равно в $S$ слову вида $a Y$ (идущему из $w$ в $\omega$ ), т.е. $X$ делится на $a$ слева.

ЗАМЕЧАНИЕ. Если $b Q=(b P)^{m}$, где $m>1$, и $b P$ не является истинной степенью, то возможно следуюшее обобщение леммы: $b Q X=X$ в $S$ тогда и только тогда, когда $X$ делится слева на $(b P)^{r} a$ для некоторого $0 \leqslant r<m$.

ТЕОРема 3.1. Для полугрупп вида $\langle a, b \mid a=b Q a\rangle$ проблема равенства слов равносильна проблеме левой делимости.

ДокАЗАТЕльство. Если $b Q$ есть истинная степень, то обе проблемы разрешимы [11]. (Мы дали второе доказательство этого факта в $\S 2$.) Пусть $b Q$ не есть истинная степень. Тогда в случае разрешимости проблемы слов мы можем выяснить, верно ли, что для данного слова $X$ имеет место равенство $b Q X=X$ в П, что равносильно делимости $X$ на $a$ слева (по лемме 3.2 ). Легко понять, что проблема делимости слева на $a$ равносильна проблеме левой делимости в П.

Тот факт, что из разрешимости проблемы левой делимости вытекает разрешимость проблемы равенства, хорошо известен (см. лемму 3.1). Теорема доказана.

Сейчас мы хотим установить факт, что проблема равенства слов в П равносильна проблеме равенства в П данного слова букве $a$.

ЛЕмма 3.3. Пусть $\Pi=\langle a, b \mid a=b Q a\rangle$, и пусть а делится слева на непустое слово $X$, причем а не равно $X$ в П. Тогда $X$ равно в П слову вида $b C_{1} \ldots b C_{r-1} b C_{r}$, zде $b C_{i} a-$ начало bQa при $1 \leqslant i<r, \quad b C_{r}-$ собственное начало $b Q a$.

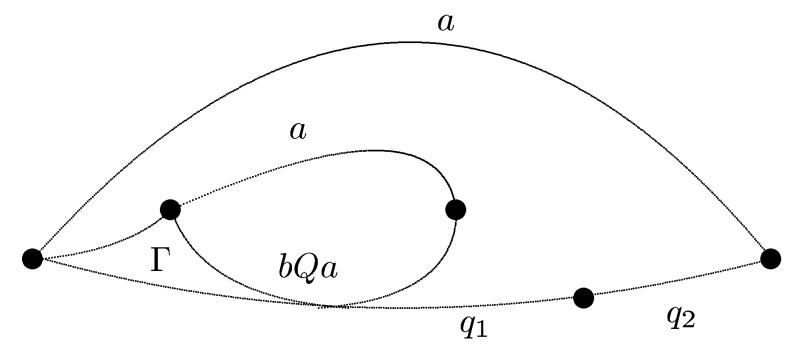

Рис. 6 
ДоКАЗАТЕЛЬСТВо. Если $a$ делится слева на $X$ и при этом $a$ не равно $X$ в П, то $a=X Y$ в П для некоторого непустого $Y$. Рассмотрим диаграмму $\Delta$, имеющую минимально возможное число клеток, граничные метки которой суть $a$ и $X^{\prime} Z$, где $X=X^{\prime}$ в П. Пусть $q_{1} q_{2}$ - нижняя граница $\Delta$, где метка $q_{1}$ есть $X^{\prime}$, а метка $q_{2}$ есть $Z$. Очевидно, что никакая клетка не может полностью примыкать к $q_{1}$ одним из своих граничных участков. Следовательно, если клетка $\pi$ примыкает к $q_{1}$, то общая часть $\pi$ и $q_{1}$ есть собственное подслово в $b Q a$. Оно должно быть началом слова $b Q a$, так как иначе в $\Delta$ можно выделить поддиаграмму $\Gamma$, отмеченную на рис. 6 , которая содержит клетки, полностью примыкаюшие к $q_{1}$ одним из своих граничных участков. Итак, слово $X^{\prime}$ есть произведение вида $b C_{1} \ldots b C_{r-1} C_{r}$, где $b C_{i}$ - начала слова $b Q$.

Пусть $1 \leqslant i<r$, и рассмотрим $i$-ю клетку, примыкающую к $q_{1}$ по слову $b C_{i}$ (рис. 7). Рассмотрим следующую, $(i+1)$-ю клетку. Она должна примыкать к пути $q_{1}$ своей стороной с меткой $b Q a$, и потому другая ее сторона примыкает к $i$-й клетке. Следовательно, $b C_{i} a$ - начало $b Q a$ при $1 \leqslant i<r$. Лемма доказана.

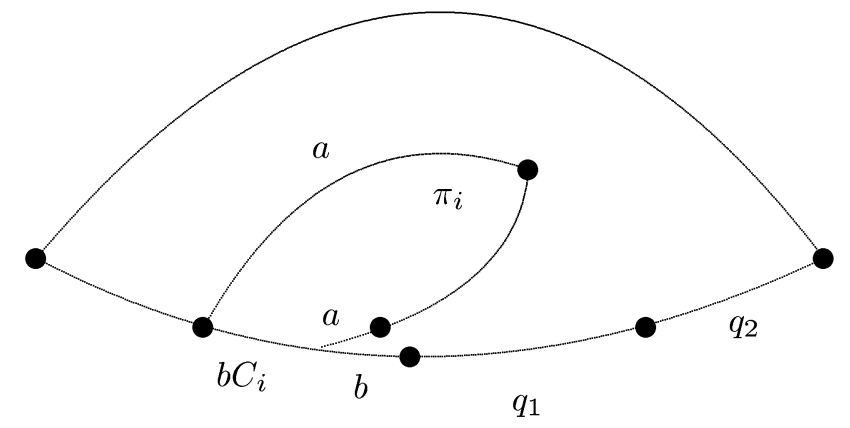

Рис. 7

ЛЕмма 3.4. Предположим, что в $\Pi=\langle a, b \mid a=b Q a\rangle$ разрешима проблема делимости слева буквы а на заданное слово $X$. Тогда в П разрешима проблема левой делимости.

ДокАЗАТЕльСтво. Нужно, располагая алгоритмом, который распознает делимость слева буквы $a$ на данное слово, научиться распознавать делимость заданного непустого слова $X$ на букву $a$ слева. Пусть дано слово $X$. Можно считать, что оно не содержит подслов вида $b Q a$. Если оно начинается с $a$, то оно делится слева на $a$. В противном случае оно начинается с $b$ и потому имеет непустое начало, на которое $a$ делится слева. Располагая соответствующим алгоритмом, выделим такое начало $V$ максимальной длины, на которое $a$ делится слева, и пусть $X \equiv V W$.

Мы можем эффективно найти, перечисляя все выводы, “частное" $Z$ от деления $a$ на $V$; если $Z$ пусто, то $X$ делится слева на $a$. Пусть $Z$ непусто; тогда ввиду леммы 3.3 существует слово вида $b C_{1} \ldots b C_{r-1} C_{r}$, описанное в условии этой леммы, равное $V$ в П. Это слово может быть найдено эффективно перечислением всех выводов. Таким образом, $X=b C_{1} \ldots b C_{r-1} b C_{r} W$ в П. Если $W$ пусто, то $X$ не делится на $a$. Пусть $W$ непусто. Допустим, что $W$ начинается с $b$. Тогда $a$ делится слева на $V b$ вопреки выбору $V$. Действительно, $a=V Z$ в П, где $Z$ - некоторое непустое слово; ясно, что $Z$ всегда делится слева на $b$. 
Итак, $W$ начинается с $a$. При этом $a$ не делится на $V a$ слева. Если $X$ делится слева на $a$, то $V a$ сравнимо слева с $a$ в П, и по следствию 1.3 остается единственная возможность, когда $V a$ делится слева на $a$. Если это имеет место, то $X$ делится слева на $V a$, а потому и на $a$. Поэтому вопрос сводится к тому, верно ли, что $b C_{1} \ldots b C_{r-1} b C_{r} a$ делится слева на $a$.

По условию $b Q a \equiv b C_{i} D_{i}(1 \leqslant i \leqslant r), b Q a \equiv b C_{r} D_{r}$ для некоторых слов $D_{1}, \ldots, D_{r}$. Тогда в П имеют место равенства

$$
\begin{aligned}
& a=b Q a=b C_{1} a D_{1}=b C_{1} b Q a D_{1}=b C_{1} b C_{2} a D_{2} D_{1}=\ldots \\
& \cdots=b C_{1} b C_{2} \ldots b C_{r-1} a D_{r-1} \ldots D_{2} D_{1}=b C_{1} \ldots b C_{r-1} b Q a D_{r-1} \ldots D_{1} \\
& \quad=b C_{1} \ldots b C_{r-1} b C_{r} D_{r} D_{r-1} \ldots D_{1} .
\end{aligned}
$$

Следовательно, делимость $b C_{1} \ldots b C_{r} a$ на $a$ слева равносильна делимости в П слева слова $b C_{1} \ldots b C_{r} a$ на слово $b C_{1} \ldots b C_{r} D_{r} \ldots D_{1}$, т.е. делимости слева $a$ на слово $D_{r} \ldots D_{1}$, а последнее свойство мы умеем эффективно распознавать. Лемма доказана.

(Мы использовали при доказательстве идеи, сходные с теми, которые применены в [11] для доказательства леммы 1.)

ТЕОРЕМА 3.2. Проблема равенства слов и проблема левой делимости слов в nолугруппе $\Pi=\langle a, b \mid a=b Q a\rangle$ равносильна проблеме равенства в П заданного слова букве а.

ДокАЗАТЕльство. Пусть последняя проблема разрешима. Допустим, что мы хотим узнать, делится ли $a$ слева на слово вида $b C_{1} \ldots b C_{r-1} b C_{r}$, где $b C_{i} a$ - начало $b Q a$ при $1 \leqslant i<r, b C_{r}$ - начало $b Q$. Положим $b Q a \equiv b C_{i} D_{i}(1 \leqslant i \leqslant r)$, $b Q a \equiv b C_{r} D_{r}$. Как мы видели в доказательстве леммы $3.4, a$ равно в П слову $b C_{1} \ldots b C_{r} D_{r} \ldots D_{1}$. Поэтому $a$ делится слева на $b C_{1} \ldots b C_{r} a$ в П тогда и только тогда, когда $D_{r} \ldots D_{1}$ делится слева на $a$.

Мы можем считать, что $b Q$ не является истинной степенью, так как в противном случае рассматриваемые проблемы разрешимы. Применим лемму 3.2. Нас интересует справедливость в П равенства $b Q D_{r} \ldots D_{1}=D_{r} \ldots D_{1}$. Домножая его слева на $b C_{1} \ldots b C_{r}$, мы получим равенство $W=b C_{1} \ldots b C_{r} b Q D_{r} \ldots D_{1}=$ $b C_{1} \ldots b C_{r} D_{r} \ldots D_{1}=a$, равносильное исходному, т.е. нам достаточно проверить, равно ли $W$ в П букве $a$.

Теперь несложно построить алгоритм, проверяющий делимость буквы $a$ на заданное слово $X$. Проводим индукцию по длине $X$. Пусть для всех слов, имеющих меньшую длину, чем $X$, мы умеем распознавать это свойство. Рассмотрим два случая.

1) $X \equiv Y b$. Проверим делимость $a$ на $Y$ слева. Если $a$ на $Y$ слева не делится, то тем более не делится и на $X$. Если же $a$ на $Y$ делится слева, то мы можем узнать, будет ли “частное" $Z$ (находимое эффективно) непустым. Если $Z$ пусто, то $Y$ равно $a$, и $a$ не делится на $X$ слева. Если же $Z$ непусто, то оно заведомо делится на $b$ слева, и потому а делится слева на $Y b=X$.

2) $X \equiv Y a$. Проверим, делится ли $a$ на $Y$ слева. Если нет, то $a$ не делится слева на $X$. Если да, то можно эффективно найти слово $b C_{1} \ldots b C_{r}$, равное в П слову 
$Y$ из леммы 3.3. Осталось узнать, делится ли $a$ слева на $b C_{1} \ldots b C_{r} a$, что мы уже умеем делать. Теорема доказана (ввиду леммы 3.4 ).

Мы можем отметить здесь еше одно следствие теоремы 3.1 , касающееся моноидов, заданных соотношением вида $a=b P b$, где $P$ - слово от $a$ и $b$. Разрешимость проблемы слов для таких полугрупп известна [3], а проблема (левой или правой) делимости открыта.

СлеДСТвИЕ 3.1. Разрешимость проблемы слов для всех полугрупп вида $\langle a, b \mid a=b Q a\rangle$ влечет разрешимость проблем левой и правой делимости для полугрупп вида $\langle a, b \mid a=b P b\rangle$.

ДоКАЗАТЕЛЬСТВо. Ясно, что достаточно говорить о левой делимости. Пусть полугруппа задана в виде $\langle a, x \mid a=x P x\rangle$, где $P=P(a, x)-$ слово от $a, x$. Заменим в слове $b P b$ каждое из вхождений буквы $x$ на $b a$. Получится слово вида $b Q a$. Пусть в $\Pi=\langle a, b \mid a=b Q a\rangle$ разрешима проблема слов. Тогда проблема левой делимости в П также разрешима по теореме 3.1. Поэтому она разрешима и в подполугруппе $S(\Pi)$ с учетом [12, лемма 5$]$. Вычисляя $S(\Pi)$ для данного случая, легко видеть, применяя алгоритм Оганесяна, что она изоморфна $\left\langle y_{1}, y_{2}\right| y_{1}=$ $\left.y_{2} P\left(y_{1}, y_{2}\right) y_{2}\right\rangle$, т.е. исходной полугруппе. Следствие доказано.

По-видимому, проблема равенства слов для полугрупп вида $\Pi=\langle a, b| a=$ $b Q a\rangle$ еше сложнее, чем проблема (левой) делимости для полугрупп, заданных при помощи $a=b P b$. Дело в том, что, как отмечалось в $\S 2$, полугруппа конщов $S(\Pi)$ не обязательно задается одним соотношением.

\section{§ 4. Проблема правой делимости}

Проблема левой делимости для полугрупп вида $\langle a, b \mid a=b Q a\rangle$ рассматривалась в ряде уже упоминавшихся работ. Однако в этих работах нет исследования проблемы правой делимости.

Как мы покажем в этом параграфе, данная проблема равносильна проблеме левой делимости и проблеме равенства слов для полугрупп, заданных при помоши соотношения $a=b Q a$.

ЛЕмма 4.1. Если непустое слово Х является правым делителем буквы а в $\Pi=\langle a, b \mid a=b Q a\rangle$, то X равно в П произведению (собственных) концов слова bQa.

ДокАЗАТЕЛЬСтво. Оговорка "собственных" несушественна, так как само слово $b Q a$ равно в П своему собственному конщу $a$.

Рассмотрим диаграмму $\Delta$, имеющую минимальное число клеток и такую, что ее граничные метки суть $a$ и $Z X^{\prime}$, где $X^{\prime}$ равно $X$ в П (ясно, что такие диаграммы имеются, если $a$ делится на $X$ справа). Если в ней нет клеток, то $X^{\prime}=a$, что является произведением конщов. Допустим, что клетки есть. Ясно, что никакая клетка не примыкает к слову $X^{\prime}$ полностью одним из своих граничных участков. Если есть клетка, примыкаюшая к $X^{\prime}$ частью слова $b Q a$, которое не есть конец этого слова, то можно применить тот же аргумент, что и в доказательстве леммы 3.3 . А именно, в диаграмме $Г$, отмеченной на рис. 8 , заведомо есть клетки, и одна из них полностью примыкает к слову $X^{\prime}$ одним из своих граничных контуров. Таким образом, $X^{\prime}$ есть произведение концов. Лемма доказана. 


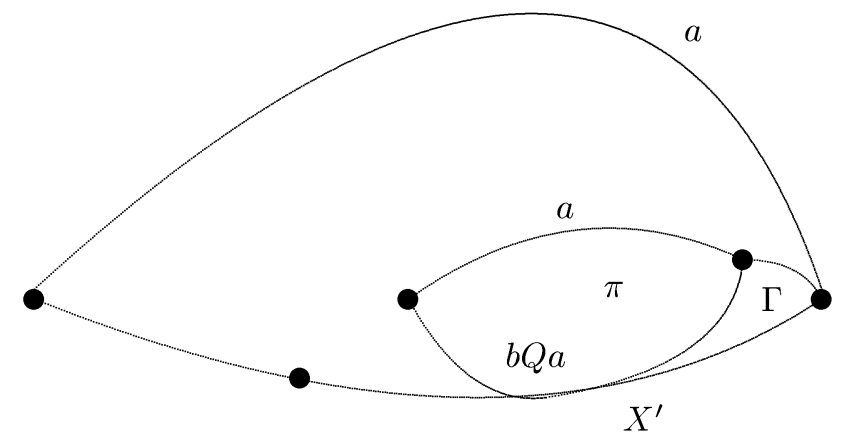

Рис. 8

ЛЕмма 4.2. Предположим, что в $\Pi=\langle a, b \mid a=b Q a\rangle$ разрешима проблема правой делимости буквы а на заданное слово. Тогда в П разрешима проблема равенства слов.

ДОКАЗАТЕЛЬСТВО. Предположим, что проблема правой делимости буквы $a$ на заданное слово разрешима. Возьмем слова $X, Y$. Нас интересует, верно ли, что $X=Y$ в П. При этом $X, Y$ должны оканчиваться на одну и ту же букву, если это условие выполнено. Так как, очевидно, можно сокращать на $b$ справа, мы можем ограничиться случаем, когда и $X$ и $Y$ оканчиваются буквой $a$.

Мы можем эффективно найти разложения вида $X \equiv X_{1} X_{2}, \quad Y \equiv Y_{1} Y_{2}$, где $X_{2}\left(Y_{2}\right)$ - конец слова $X(Y)$ максимальной длины такой, что $a$ делится на него справа в П. Проверим, что равенство $X$ и $Y$ в П влечет равенство $X_{1}=Y_{1}$ в П (а потому и равенство $X_{2}=Y_{2}$, так как равенства можно сокращать слева). Заметим, что $X_{2}$ и $Y_{2}$ непустые.

Предположим, что $X_{1} \neq Y_{1}$ в П. Тогда, поскольку $X_{1}$ и $Y_{1}$ в П сравнимы слева, мы имеем ввиду следствия 1.3 , что либо $X_{1}$ делится слева на $Y_{1}$, либо наоборот.

Допустим, что $X_{1}$ делится на $Y_{1}$ слева. Тогда $X_{1}=Y_{1} W$ для некоторого слова $W$, причем $W$ непусто. Ясно, что $X_{1}$ и $W$ оканчиваются одной и той же буквой $c \in\{a, b\}$. Так как $X_{1} X_{2}=Y_{1} Y_{2}$ в $\Pi$, то $Y_{1} W X_{2}=Y_{1} Y_{2}$, т.е. $W X_{2}=Y_{2}$ в $\Pi$. Однако тогда $a$ делится справа на $W X_{2}$, а потому и на $c X_{2}$. Но $c X_{2}-$ конец слова $X_{1} X_{2}$, так как $c$-последняя буква слова $X_{1}$. Это противоречит выбору слова $X_{2}$.

Слова $X_{1}$ и $Y_{1}$ в сумме имеют меньшую длину, чем слова $X$ и $Y$. Из индуктивных соображений можно считать, что мы умеем выяснять, верно ли что $X_{1}$ и $Y_{1}$ равны в П. Осталось выяснить, верно ли, что $X_{2}$ и $Y_{2}$ равны в П (заметим, что наиболее интересен случай, когда $X=X_{2}, Y=Y_{2}$, т.е. оба слова - правые делители $a)$. Однако ввиду леммы 4.1 слова $X_{2}, Y_{2}$ принадлежат полугруппе $S(\Pi)$, порожденной в П непустыми концами слова $b Q a$. Согласно следствию 2.2 проблема слов в $S(\Pi)$ разрешима. Остается проверить, равны ли слова $X_{2}, Y_{2}$ в $S(\Pi)$, что и дает ответ на наш вопрос. Лемма доказана.

Лемма 4.3. Пусть в $\Pi=\langle a, b \mid a=b Q a\rangle$ разрешима проблема правой делимости буквы а на заданное слово. Тогда в П разрешима проблема правой делимости.

ДокАЗАТЕЛЬСТво. Пусть даны слова $X, Y$. Мы хотим узнать, делится ли $X$ на $Y$ справа. Очевидным образом можно считать, что $X$ и $Y$ оканчиваются на $a$. 
Пусть $X \equiv X_{1} X_{2}, \quad Y \equiv Y_{1} Y_{2}$, где $X_{2}\left(Y_{2}\right)$ - максимальный конец слова $X(Y)$ такой, что $a$ делится на него справа в П. Эти разложения могут быть найдены эффективно.

Если $Y_{1}$ пусто, то а делится справа на $Y$, и потому $X$ делится справа на $Y$. Считаем $Y_{1}$ непустым. Если $X$ делится на $Y$ справа, то $X=Z Y$ в П для некоторого слова $Z$. Поскольку $X_{1} X_{2}=Z Y_{1} Y_{2}$ в П и $X_{2}, Y_{2}$ - максимальные концы слов, которые являются правыми делителями $a$, то согласно доказательству леммы 4.2 должны быть справедливы равенства $X_{1}=Z Y_{1}, X_{2}=Y_{2}$ в П. Последнее условие мы можем проверить эффективно. Если оно не выполнено, то $X$ не делится на $Y$ справа. Если оно выполнено, то $X$ делится справа на $Y$ в том и только том случае, когда $X_{1}$ делится справа на $Y_{1}$. Действительно, мы установили, что делимость $X$ на $Y$ справа влечет делимость $X_{1}$ на $Y_{1}$ справа. Обратно, пусть $X_{1}=W Y_{1}$ в П. Тогда $X=X_{1} X_{2}=W Y_{1} X_{2}=W Y_{1} Y_{2}=W Y$ в $\Pi$, так как $X_{2}$ и $Y_{2}$ равны в $\Pi$.

Теперь нам осталось выяснить вопрос о делимости справа $X_{1}$ на $Y_{1}$, но эти слова по сумме длин меньше, чем $X$ и $Y$, и доказательство завершается при помоши индукции по сумме длин слов $X$ и $Y$ Лемма доказана.

ЛЕмма 4.4. Пусть в $\Pi=\langle a, b \mid a=b Q a\rangle$ разрешима проблема левой делимости. Тогда в П разрешима проблема правой делимости буквы а на заданное слово.

ДокАЗАТЕЛЬСТво. Пусть требуется выяснить, делится ли $а$ справа на заданное слово $X$. Проводим индукцию по длине слова $X$. Пусть $c \in\{a, b\}-$ первая буква слова $X$ и $X \equiv c Y$. Ясно, что $Y$ можно считать непустым. Мы вначале проверим, делится ли $a$ на $Y$ справа. Допустим, что да, так как в противном случае $a$ не делится справа на $X$. Будем считать, что $a=V Y$ в П. Нам достаточно теперь установить следующий вспомогательный факт: буква а делится на сY справа тогда и только тогда, когда существует конец, сDа слова bQа такой, что $Y$ делится на Dа слева.

Поскольку мы имеем конечное число таких конщов и для каждого из них проверка этого условия возможна ввиду разрешимости проблемы левой делимости, то нам достаточно доказать указанный факт.

Предположим, что $Y$ делится на $D a$ слева; положим $Y=D a Z$ в П. Так как $c D a-$ конец слова $b Q a$, можно положить $b Q a \equiv W c D a$. Имеем равенства в П

$$
a=V Y=V D a Z=V D b Q a Z=V D W c D a Z=V D W c Y=V D W X,
$$

т.е. $a$ делится на $X$ справа.

Докажем обратное. Пусть $a$ делится справа на $c Y$. Рассмотрим диаграмму $\Delta$, имеющую минимально возможное число клеток, такую, что ее граничные метки имеют вид $a$ и $U c Y$ для некоторого $U$. Так как $Y$ непусто, ребро нижней границы, имеющее метку $c$, не принадлежит верхней границе. Следовательно, к нему примыкает некоторая клетка. Если это клетка с верхней граничной меткой $b Q a$ и нижней граничной меткой $a$, примыкающей к $c$, то ее можно удалить, получая диаграмму с меньшим числом клеток; при этом нижняя граничная метка полученной диаграммы имеет вид $U b Q \cdot c Y$.

Следовательно, клетка, примыкающая к $c$, примыкает своей нижней границей, имеющей метку $b Q a$. Если ее последнее ребро с меткой $a$ примыкает к $c$ (рис. 9), то 


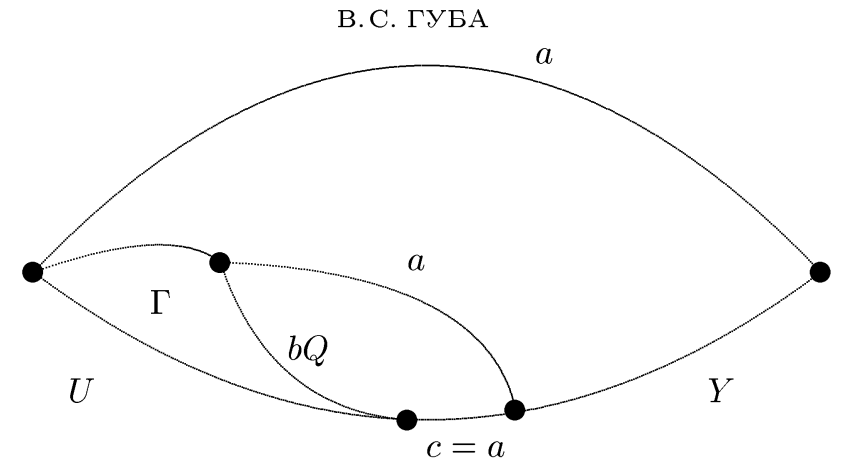

Рис. 9

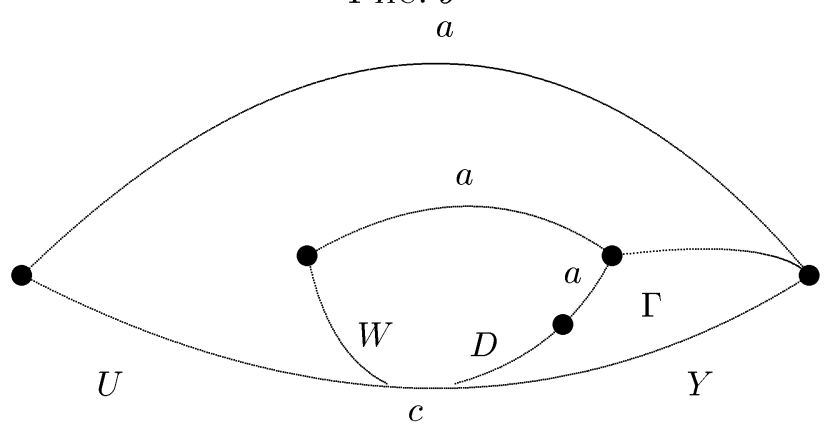

Рис. 10

можно удалить эту клетку вместе с отмеченной поддиаграммой $\Gamma$, уменьшая число клеток.

Наконец, пусть клетка примыкает к $c$ так, что не последнее ее ребро примыкает к $c$ (рис. 10). В этом случае $b Q a \equiv W c D a$ при некоторых $D, W$, и из рассмотрения поддиаграммы $Г$ видно, что $Y$ делится на $D a$ слева, что и требовалось установить. Лемма доказана.

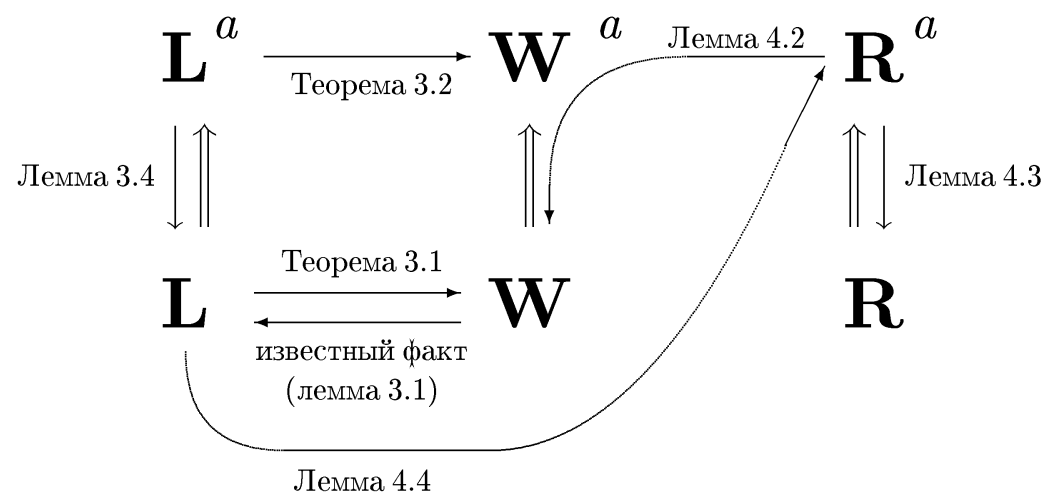

Рис. 11

Tеорема 4.1. Для полугруппы $\Pi=\langle a, b \mid a=b Q a\rangle$ равносильны следующие алгоритмические проблемы: проблема равенства слов, проблема левой 
делимости, проблема правой делимости. Все эти проблемы равносильны проблеме равенства данного слова букве а.

ДокАЗАТЕЛЬСТво. Эта теорема следует из теоремы 3.1 , лемм 4.2-4.4 и теоремы 3.2 .

Для удобства изобразим (рис. 11) схему (где $\mathbf{W}, \mathbf{L}, \mathbf{R}$ - проблемы слов, левой и правой делимости; $\mathbf{W}^{a}$ - проблема равенства заданного слова букве $a ; \mathbf{L}_{a}\left(\mathbf{R}_{a}\right)$ проблема левой (правой) делимости данного слова на $a ; \mathbf{L}^{a}\left(\mathbf{R}^{a}\right)$ - проблема левой (правой) делимости буквы $a$ на заданное слово). Отметим, что проблема $\mathbf{R}_{a}$ тривиальна, и мы ее не включаем в схему. Очевидные связи изображены стрелкой $\Longrightarrow$. Мы не включаем также $\mathbf{L}_{a}$, поскольку она очевидныг образом равносильна $\mathbf{L}$.

СлЕДСТВИЕ 4.1. Для полугрупп вида $\Pi=\langle a, b \mid a=b Q a\rangle$, удовлетворяющ,их одному из условий (1)-(5) (см. теорему 2.2), разрешима проблема правой делимости.

Это сразу вытекает из теорем 2.2 и 4.1.

В заключение данного параграфа хотелось бы задать следуюший интересный

Вопрос. Существует ли такая полугруппа П вида $\langle a, b \mid a=b Q a\rangle$ и такое слово $X$, что для любого натурального $k$ слово $X$ делится в П слева на $a^{k}$ ?

Нам представляется, что ответ на него поможет прояснить вопрос о разрешимости рассматриваемых алгоритмических проблем в полугруппах вида $\langle a, b| a=$ $b Q a\rangle$.

\section{§ 5. О свойствах полугрупп, заданных соотношениями без односторонних циклов}

Данный параграф тесно связан с изложенным выше, так как проблемы равенства и делимости для полугрупп с одним определяюшим соотношением сведены к случаю двухбуквенного алфавита и несократимого с одной стороны соотношения [7].

Мы будем изучать свойства полугрупп без левых циклов. (Очевидные аналоги установленных фактов имеют место и для полугрупп без правых циклов.) Нас будут интересовать алгебраические свойства таких полугрупп.

Пусть $\left\langle x_{1}, \ldots, x_{m} \mid A_{i}=B_{i}(1 \leqslant i \leqslant k)\right\rangle$ - копредставление без левых циклов (очевидно, $k<m$ ). Пусть $S$ - полугруппа с единищей, задаваемая им. Для удобства мы присоединим к $S$ нулевой элемент 0 . Получившуюся полугруппу с присоединенным нулем обозначим через $S_{0}$.

Следуюшую лемму можно извлечь из работы [12].

ЛЕмма 5.1. Пусть П - полугруппа с единицей, заданная копредставлением без левьх ииклов. Тогда для любых слов $x, y$ пересечение $x \Pi \cap y \Pi$ либо пусто, либо равно $z \Pi$, где $z$-некоторое слово.

ДокАЗАТЕЛЬСтво. Если $x, y$ не сравнимы в П слева, то $x \Pi \cap y \Pi=\varnothing$. Допустим, что $x, y$ сравнимы в П слева. По лемме $3[12] z=x f(x, y)=y f(y, x)$ в П (см. там же необходимые обозначения). Если $w \in x \Pi \cap y \Pi$, то $w=x u=y v$ в П для некоторых $u, v$, и ввиду [12, лемма 2] $w \in x f(x, y) \Pi=z \Pi$. Отсюда очевидно, что $x \Pi \cap y \Pi=z \Pi$. Лемма доказана. 
СлЕДСТВИЕ 5.1. Если П - полугруппа с единицей, заданная копредставлением без левых ииклов, то в полугруппе $\Pi_{0}$ (с присоединенным нулем) пересечение главных правых идеалов - главный правый идеал.

Отметим очевидный факт, что для любых элементов $x, y \in \Pi_{0}$ условие $x \Pi_{0}=$ $y \Pi_{0}$ равносильно $x=y$. Таким образом, $\Pi_{0}$ находится во взаимно однозначном соответствии с множеством своих главных правых идеалов.

Можно определить операцию + на $\Pi_{0}$, полагая по определению $(x+y) \Pi_{0}=$ $x \Pi_{0} \cap y \Pi_{0}$ (для любых $x, y \in \Pi_{0}$ такой элемент определен однозначно). Ясно, что $x+y=y+x, x+x=x$ для любых $x, y \in \Pi_{0}$, т.е. данная операция преврашает $\Pi_{0}$ в (верхнюю) полурешетку (т.е. коммутативную полугруппу идемпотентов). С этой операцией естественно связано отношение частичного порядка $\succeq$, где $x \succeq y$ по определению означает $x=x+y$, что равносильно условию $x \Pi_{0} \subseteq y \Pi_{0}$. Это означает, что либо $x=0$, либо $x$ делится на $y$ слева.

Каковы свойства данной полурешетки?

ТЕОРема 5.1. Пусть П - полугруппа с единицей, заданная копредставлением без левых ииклов. Тогда определенная выше полурешетка $\left\langle\Pi_{0},+\right\rangle$ удовлетворяет следующему условию: для любых $a_{1}, \ldots, a_{r} \in \Pi_{0}$, әде $r \geqslant 2$, найдутся $a_{i}, a_{j}(i \neq j)$ mакие, ито $a_{1}+\cdots+a_{r}=a_{i}+a_{j}$.

Заметим, что далеко не каждая полугруппа удовлетворяет заключению следствия 5.1 и, тем более, теоремы 5.1. Эта теорема накладывает весьма сильные алгебраические ограничения на полугруппу. Говоря теперь уже об идеалах полурешетки, мы можем так переформулировать теорему: любой конечно порожденньй идеал полурешетки порождается двумя элементами. Интересно либо найти какие-то еше алгебраические условия такого сорта, необхдимо выполняюшиеся в полугруппах без левых циклов, либо доказать, что полугруппа с некоторым набором чисто алгебраических свойств обладает копредставлением без левых циклов (хотя бы в данном множестве образуюших).

ДОКАЗАТЕЛЬСТВо.Достаточно ограничиться рассмотрением случая трех элементов: $a_{1}, a_{2}, a_{3}$. Пусть $X_{1}, X_{2}, X_{3}$ - слова над алфавитом порождаюших П, соответствуюшим ее копредставлению без левых циклов. Если какие-либо два слова из трех не сравнимы в П слева, то их сумма, как и сумма всех трех слов, равна нулю. Поэтому далее предположим, что все рассматриваемые слова попарно сравнимы в П слева.

Пусть слова $X, Y$ сравнимы слева в П. Тогда определены слова $f(X, Y), f(Y, X)$ и имеется единственная диаграмма, осушествляюшая равенство $X f(X, Y)=$ $Y f(Y, X)$, имеюшая минимально возможное число клеток. Назовем ее диаграммой сравнимости слева слов $X, Y$ или просто диаграммой сравнимости.

Выберем теперь диаграммы сравнимости слева любой из трех пар вида $X_{i}, X_{j}$ $(i \neq j)$. Именно, пусть $\Delta_{i}$ - диаграмма, осуществляющая равенство в П слов $X_{i+1} f\left(X_{i+1}, X_{i+2}\right)$ и $X_{i+2} f\left(X_{i+2}, X_{i+1}\right)$ (здесь и далее действия над нижними индексами производятся по модулю 3 , а сами индексы принимают значения от 1 до 3). Наша задача - установить следующий факт: при некотором $i$ одно из слов $f\left(X_{i}, X_{i-1}\right), f\left(X_{i}, X_{i+1}\right)$ является началом другого; проверим, что из этого будет следовать утверждение теоремы. 
Без ограничения обшности пусть $f\left(X_{i}, X_{i-1}\right) \equiv f\left(X_{i}, X_{i+1}\right) W$. Тогда в П имеем равенства

$$
X_{i-1} f\left(X_{i-1}, X_{i}\right)=X_{i} f\left(X_{i}, X_{i-1}\right)=X_{i} f\left(X_{i}, X_{i+1}\right) W=X_{i+1} f\left(X_{i+1}, X_{i}\right) W
$$

т.е. элемент $X_{i-1} f\left(X_{i-1}, X_{i}\right)=X_{i} f\left(X_{i}, X_{i-1}\right)$, порождающий главный правый идеал $X_{i-1} \Pi_{0} \cap X_{i} \Pi_{0}$, делится в П слева на $X_{i+1}$. Это дает включение $X_{i-1} \Pi_{0} \cap$ $X_{i} \Pi_{0} \subseteq X_{i+1} \Pi_{0}$, приводящее к равенству $X_{1}+X_{2}+X_{3}=X_{i-1}+X_{i}$.

Осталось доказать требуемый факт, что будет сделано от противного. Рассмотрим "минимальньй" контрпример, имея в виду минимальное суммарное число клеток в диаграммах $\Delta_{1}, \Delta_{2}, \Delta_{3}$, при фиксированном числе клеток - минимальную сумму длин шести слов вида $f\left(X_{i}, X_{j}\right)(i \neq j)$ и при фиксированных значениях предыдуших параметров - минимальную сумму длин трех слов $X_{i}$. Ввиду очевидного равенства $1+x=x$ все слова $X_{i}$ можно считать непустыми.

Поскольку мы рассматриваем контрпример, все шесть слов вида $f\left(X_{i}, X_{j}\right)$ непусты. Проверим, что для любого $i$ слова $f\left(X_{i}, X_{i-1}\right)$ и $f\left(X_{i}, X_{i+1}\right)$ начинаются с разных букв. Если они начинались бы с одной и той же буквы $x$, то слово $X_{i}$ можно было бы заменить на $X_{i} x$. При этом $f\left(X_{i}, X_{i-1}\right) \equiv x f\left(X_{i} x, X_{i-1}\right)$, $f\left(X_{i}, X_{i+1}\right) \equiv x f\left(X_{i} x, X_{i+1}\right)$. Отсюда следует, что контрпример можно уменьшить, так как диаграммы не изменяются, а два из шести слов вида $f\left(X_{i}, X_{j}\right)$ уменьшают длину на единицу.

Каждой диаграмме естественным образом можно сопоставить путь в левом графе. Именно, делая обход начальной вершины диаграммы по часовой стрелке на малом расстоянии, будем отмечать, в какие из клеток мы попадаем. Каждой клетке соответствует определяющее соотношение, а потому и ребро левого графа. Такие ребра образуют требуемьй путь.

Теперь рассмотрим пути $p_{1}, p_{2}, p_{3}$ в левом графе, соответствующие диаграммам $\Delta_{1}, \Delta_{2}, \Delta_{3}$. Их произведение - путь $p$ с одной и той же начальной и конечной вершиной. Допустим вначале, что этот путь состоит из одной вершины. Это в точности значит, что все слова $X_{i}$ начинаются с одной и той же буквы (напомним, что все слова $X_{i}$ непусты). Легко понять, что при замене каждого из слов $X_{i}$ на слово без начальной буквы, мы вновь имеем контрпример, что противоречит минимальности выбранного контрпримера. Следовательно, путь $p$ непуст, и ввиду отсутствия циклов в левом графе находим, что $p$ содержит пару подряд проходимых взаимно обратных ребер $е$ и $e^{-1}$.

Легко понять, что клетки, соответствующие этим ребрам левого графа, находятся в разных диаграммах. Действительно, если они содержатся в одной и той же диаграмме $\Delta_{i}$, то они не могут образовывать зеркальную пару ввиду минимальности числа клеток в $\Delta_{i}$. Если они расположены, как на рис. 12 , то в $\Delta_{i}$ имеется поддиаграмма, метки контуров которой начинаются с одной буквы, но соответствуют различным ребрам диаграммы. Из условия минимальности диаграммы и отсутствия циклов в левом графе сразу следует, что начальные ребра контуров диаграммы равны, что приводит к противоречию.

Итак, будем считать, что ребру $е$ соответствует клетка $\pi$ в $\Delta_{1}$, а ребру $e^{-1}$ клетка $\pi^{\prime}$ в $\Delta_{2}$. Для удобства склеим начало нижнего контура диаграммы $\Delta_{1}$, имеюшее метку $X_{3}$, с началом верхнего контура диаграммы $\Delta_{2}$, имеюшим ту же метку (рис. 13). 


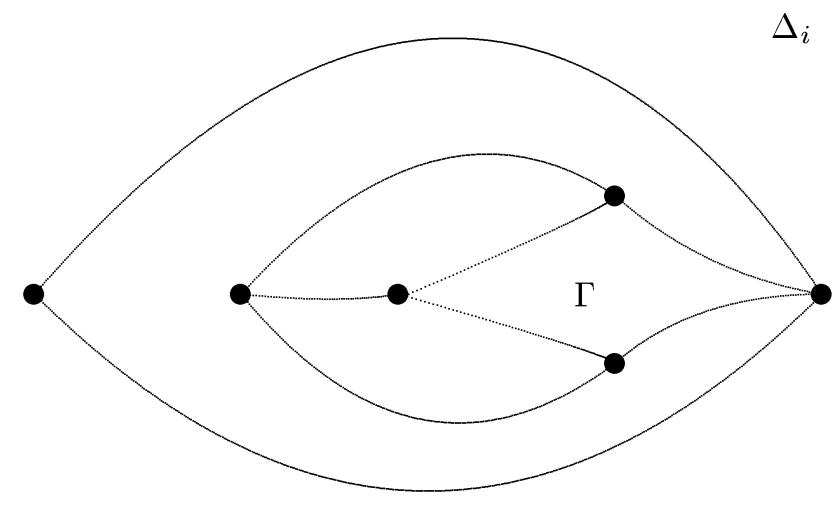

Рис. 12

Пусть $t$ - путь, по которому произведена склейка. Нижний контур клетки $\pi$ обозначим через $q_{1}$, а верхний контур $\pi^{\prime}$ - через $q_{2}$. Их максимальное общее начало $q$ непусто и является началом пути $t$.

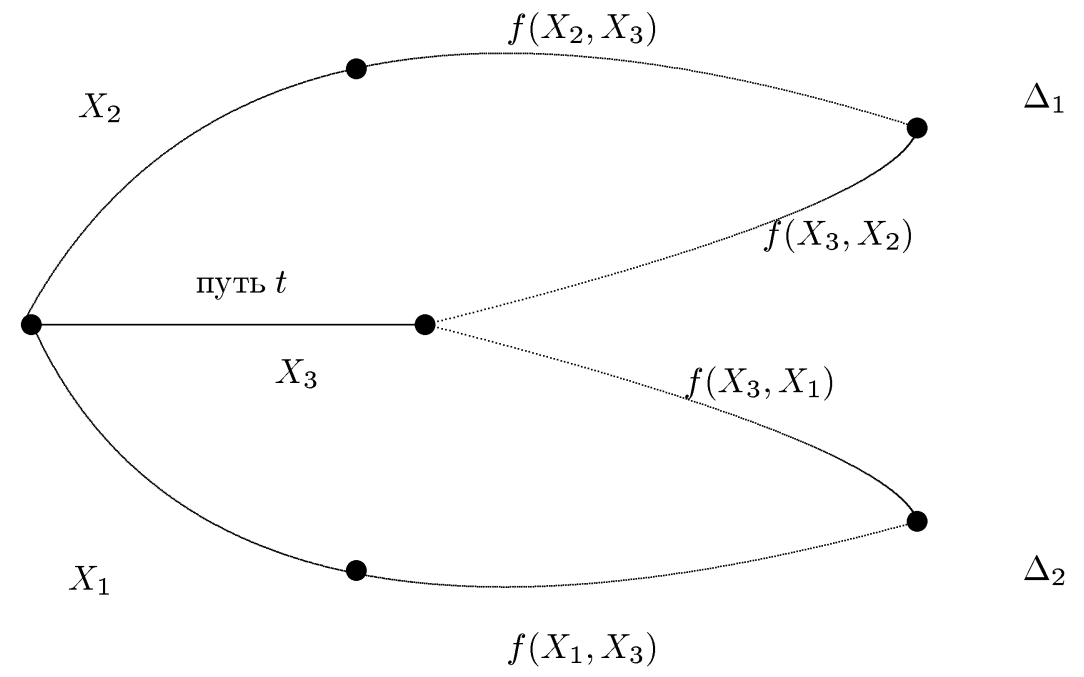

Рис. 13

Рассмотрим пары клеток $\pi_{1}, \pi_{2}$ со следующими свойствами:

$-\pi_{j}$ лежит в $\Delta_{j}(j=1,2)$;

- метка $U(V)$ верхнего контура $\pi_{1}\left(\pi_{2}\right)$ графически равна метке нижнего контура $\pi_{2}\left(\pi_{1}\right)$;

- нижний контур клетки $\pi_{1}$ имеет вид $s s_{1}$, а верхний контур $\pi_{2}-$ вид $s s_{2}$, где $s-$ непустое максимальное начало этих контуров;

- путь $s-$ подпуть в $t$. 
Ясно, что такие пары имеются. Рассмотрим, сохраняя обозначения, такую из них, для которой подпуть $s$ в $t$ расположен как можно правее.

Рассмотрим случай, когда пути $s_{1}, s_{2}$ пусты. Покажем, как уменьшить контрпример. Исключим клетки $\pi_{1}, \pi_{2}$ из диаграмм $\Delta_{1}, \Delta_{2}$ соответственно, получая диаграммы $\Delta_{1}^{\prime}, \Delta_{2}^{\prime}$, а вместо слова $X_{3}$ рассмотрим слово $X_{3}^{\prime}$, получаемое из $X_{3}$ заменой подслова $V$ на слово $U$ таким образом, что вхождение слова $V$ в $X_{3}$ получается из вхождения пути $s$ с меткой $V$ в путь $t$ с меткой $X_{3}$. Слова $X_{1}, X_{2}, X_{3}^{\prime}$ приводят к трем диаграммам сравнимости, которые суть в точности $\Delta_{1}^{\prime}, \Delta_{2}^{\prime}, \Delta_{3}$. После описанных действий мы вновь имеем контрпример, но три новых диаграммы имеют на две клетки меньше, чем старые. Это дает противоречие.

Теперь рассмотрим случай, когда пути $s_{1}, s_{2}$ непусты (напомним, что их метки одинаковы). Пусть $v$-конецпути $s$, и пусть $e_{j}-$ первое ребро пути $s_{j}(j=1,2)$. Для начала допустим, что $v$ - конец пути $t$. Мы утверждаем, что $e_{1}\left(e_{2}\right)$ принадлежит нижней (верхней) границе диаграммы $\Delta_{1}\left(\Delta_{2}\right)$. Предполагая, что $e_{1}$ не обладает этим свойством, мы можем выделить поддиаграмму в $\Delta_{1}$ с той же верхней границей, взяв в качестве нижней гранищы некоторый путь, начинающийся с $s e_{1}$. Легко понять, что эта поддиаграмма имеет меньше клеток, чем $\Delta_{1}$, но метки ее контуров по-прежнему начинаются словами $X_{2}, X_{3}$. Тем самым мы получаем противоречие со свойством диаграмм сравнимости (см. их определение вьше). Рассуждения для $e_{2}$ аналогичны. В результате оказывается, что $f\left(X_{3}, X_{2}\right)$ и $f\left(X_{3}, X_{1}\right)$ начинаются одной и той же буквой, которая есть метка ребер $e_{1}$ и $e_{2}$. Это противоречит тому, что для любого $i$ слова $f\left(X_{i}, X_{i-1}\right)$ и $f\left(X_{i}, X_{i+1}\right)$ начинаются с разных букв.

Осталось рассмотреть последний случай, когда конец пути $s$ не совпадает с концом пути $t$. Ребра $e_{1}, e_{2}$ различны по определению пути $s$. Они имеют одну и ту же метку $x$. Пусть $e$ - ребро пути $t$ с меткой $y$, выходящее из вершины $v$. Двигаясь вблизи $v$ от ребра $e_{1}$ к ребру $е$ по часовой стрелке, мы можем рассмотреть соответствующий путь в левом графе из $x$ в $y$ подобно тому, как это делалось ранее. Аналогично, двигаясь вблизи $v$ в том же направлении от $e$ к $e_{2}$, мы получим путь в левом графе из $y$ в $x$. Произведение $p$ этих путей непусто. Оно является петлей из $x$ в $x$, и потому путь $p$ имеет два взаимно обратных ребра, проходимых подряд. Этим ребрам соответствуют клетки, одна из которых лежит в $\Delta_{1}$, а другая - в $\Delta_{2}$, что доказывается так же, как это уже делалось. Полученные клетки образуют пару, обладающую всеми свойствами, которые отмечались при выборе клеток $\pi_{1}, \pi_{2}$,

однако расположены они правее, что противоречит правилу выбора клеток $\pi_{1}, \pi_{2}$. Опять пришли к противоречию. Теорема доказана.

\section{Список литературы}

1. Адян С.И. О вложимости полугрупп в группы // ДАН СССР. 1960. Т. 133. № 2. C. $255-257$.

2. Адян С. И. К проблеме тождества в ассоциативных системах специального вида // ДАН СССР. 1960. Т. 135. №6.

3. Адян С.И. Определяющие соотношения и алгоритмические проблемы для групп и полугрупп // Тр. МИАН. 1966. Т. 85. С. 1-124.

4. Адян С.И. О преобразованиях слов в полугруппе, заданной системой определяющих соотношений // Алгебра и логика. 1976. Т. 15. №6. С. 611-621.

5. Адян С.И. К проблеме делимости для моноидов, заданных одним соотношением // Матем.заметки. 1994. Т. 55. № 1. С. 3-9. 
6. Адян С.И., Оганесян Г. У. К проблемам равенства и делимости в полугруппах с одним определяющим соотношением // Изв. АН СССР. Сер.матем. 1978. Т. 42. № 2. С. 219-225.

7. Адян С. И., Оганесян Г. У. О проблемах равенства и делимости для полугрупп с одним соотношением // Матем. заметки. 1987. Т. 41. № 3. С. 412-421.

8. Кашинцев E. В. Графы и проблема слов для конечно-представленных полугрупп // Уч. зап. Тульского пед. ин-та. 1970. № 2. С. 290-302.

9. Линдон P., Шупп П. Комбинаторная теория групп. М.: Мир, 1980.

10. Михайлова K. А. Проблема вхождения для прямых произведений групп // ДАН СССР. 1958. T. 119. C. $1103-1105$.

11. Оәанесян Г.У. О проблемах равенства и делимости в полугруппах с определяющим соотношением вида $a=b A$ // Изв. АН СССР. Сер.матем. 1978. Т. 42. № 3. С. 602-612.

12. Оъанесян Г. У. О полугруппах с одним соотношением и полугруппах без циклов // Изв. АН СССР. Сер.матем. 1982. Т. 46. №1. С. 88-94.

13. Оганесян Г. У. О проблеме изоморфизма для полугрупп с одним определяющим соотношением // Матем. заметки. 1984. Т. 35. № 5. С. 685-691.

14. Оганесян Г.У. Делимость и равенство слов в полугруппах с одним соотношением // III Всесоюзный симпозиум по теории полугрупп. Свердловск, 1988.

15. Ольианский А. Ю. Геометрия определяющих соотношений в группах. М.: Наука, 1989.

16. Саркисян $O . A$. Некоторые соотношения между проблемами тождества и делимости в группах и полугруппах // Изв. АН СССР. Сер.матем. 1979. Т. 43. № 4. С. 909-921.

17. Саркисян $O . A$. О проблемах тождества и делимости в полугруппах и группах без циклов // Изв. АН СССР. Сер.матем. 1981. Т. 45. №6. С. 1424-1441.

18. Baumslag G. Positive one-relator groups // Trans. Amer. Math. Soc. 1971. V. 156. P. 165-183.

19. Benois M. Parties rationnelles du groupe libre // C.R. Acad. Sci. Paris. Sér. A. 1969. V. 269. P. $1188-1190$.

20. Higgins P. M. Techniques of Semigroup Theory. New York: Oxford University Press, 1992.

21. Howie J., Pride S. J. The word problem for one-relator semigroups // Math. Proc. Camb. Phil. Soc. 1986. V. 99. P. 33-44.

22. Magnus $W$. Das Identitäts - Problem für Gruppen mit einer definierenden Relation // Math. Ann. 1932. V. 106. P. 295-307.

23. Meakin J., Sapir M. Congruences of free monoids and submonoids of polycyclic monoids // J. Austral. Math. Soc. Series A. 1993. V. 54. P. 236-253.

24. Remmers J. H. On the geometry of semigroup presentations // Advances in Math. 1980. V. 36. № 3. P. 283-296.

25. Rips E. Subgroups of small cancellation groups // Bull. London Math. Soc. 1982. V. 14. № 1. P. 45-47. 